\title{
Conjugaison par tranches $(*)$.
}

\author{
MICHEI, VOLLE (**) (Limoges, France)
}

Résumé. - Etant donné un opérateur de fermeture on s'intéresse aux fonctions réelles dont les tranehes sont fermées. Ces fonstions, stables por passage à l'enveloppe supérieure, définissent une régularisation. En décomposant l'opérateur de fermeture relativement à une polarité, on introduit une (bi) conjugaison des fonctions réelles $f$ de sorte que la biconjuguée de $f$ coïncide avec sa régularisée. La théorie s'applique à diverses formes de dualité quasiconvexe et à la programmation mathématique en général.

Summary. - We are interested here by extended real valued functions whose level sets are closed with respect to a given closure operator. This class of functions is closed under pointwise suprema so that a regularization can be defined. By using the notion of polarity we decompose the closure operator and introduce a (bi) conjugation for the real extended valued functions $f$ such that the biconjugate of $f$ is just the regularized of $f$. We apply this theory to many forms of quasiconvex dualities and to mathematical programming in the general form.

\section{Introduction.}

Etant donné un opérateur de fermeture on s'intéresse aux fonctions réelles dont les tranches sont fermées. Ces fonctions sont d'un usage fréquent en mathématiques, citons par exemple les fonctions monotones, s.c.i., quasiconrexes, les $V$-morphismes de treillis complets (Th. II.2.1), etc.

On utilise la décomposition de l'opérateur de fermeture relativement à une polarité (à savoir une application entre espaces d'ensembles qui transforme les réunions en intersections) pour définir une (bi) conjugaison des fonctions réelles (et, plus généralement, des multiapplications) de sorte que la biconjuguée d'une fonctions réelle $f$ soit la plus grande fonction à tranches fermées qui minore $f$.

Nous remarquons (Th. I.2.1) que cette conjugaison s'interprête au sens de Fenchel généralisé (Moreav [29], [30]) au cas d'une fonction de couplage ne prenant que les valeurs 0 et $-\infty$. Inversement, toute conjugaison au sens de Fenchel généralisé peut être décrite (Th. I.2.4) par la conjugaison relative à une polarité bien choisie.

La notion de polarité dont nous avons besoin a été utilisée, plus ou moins explicitement, par ORE [32], [33], BIRKHoFF [4] et, plus récemment, par EvERs, MAAREN [16], DolfCkI, Araoz, Edmonds, Griffin [1]. Cette notion s'étend aux

(*) Entrata in Redazione il 28 settembre 1983; versione riveduta il 13 marzo 1984.

(**) Département de Mathématique, U.E.R. des Sciences. 123 rue Albert Thomas, 87060 Limoges Cedex. 
treillis complets de manière évidente et on sait (Everetr [15], Pickert [38]) qu'elle y est équivalente à celle de correspondance de GaLoIs ([4], [32]). L'intérêt de cette extension est que, outre les treillis complets $\left(2^{x}, c\right)$, on considère aussi les treillis complets $\overline{\mathbb{R}}^{X}$ munis de l'ordre produit.

L'étude des fonctions dont les tranches sont des cônes "evenly" convexes (FENCHEL [18]) nous permet de retrouver l'approche de PASSY et PRISMaN ([34], [35]) et de compléter certains de leurs résultats (Th. IIr.1.2); signalons sur ce point le travail récent de J. E. MARTINEZ-LeGAz [27] dont l'auteur vient d'avoir connaissance. De même, pour les fonctions dont les tranches sont des épigraphes de fonctions convexes s.e.i. on éméliore (Th. III.3.10) un résultat de Flachs et PoLLATSCHEK ([19], Th. 2).

En fait, de nombreuses conjugaisons introduites en quasiconvexité (GREENBERGPierskalda [22], Singer [40], AtTeia, El Qortobi [2], [13], Crouzetx [8], etc.) s'apparentent à des conjugaisons relatives à des polarités; ceci permet de caractériser par des propriétés de tranches les fonctions conjuguées (Th. III.2.1, III.3.1, III.3.7, III. 4.1, etc.).

Pour les fonctions marginales rencontrées en programmation mathématique, on introduit une polarité très maniable pour laquelle Jes tranches de ces fonctions sont fermées (Prop. IV.1.1) ce qui en facilite l'étude (Cor. IV. 1.2, Prop. IV. 1.3).

On introduit enfin une notion de programme dual d'un programme donné relativement à une perturbation et à une polarité. Les solutions du dual sont décrites à l'aide de la notion de $\Delta$-quasidifférentiel préalablement définie (Prop. IV.2.1). Nous considérons un lagrangien dont les points selles coïncident avec les couples solutions des problèmes primal et dual (Th. IV.2.3). Cela nous fournit par exemple un lagrangien pour la dualité quasiconvexe s.c.i. de Orouzerx [7] (Th. IV.4.1). De même, nous récupérons le lagrangien introduit par PASSY et PRISMAN dans [35].

Notre démarche permet de donner de nouveaux exemples de problèmes d'optimisation en dualité et de nouveux lagrangiens. Ainsi, on montre que pour une certaine polarité, le problème d'optimisation posé par la mesure de Jordan extérieure d'un ensemble admet pour dual la mesure intérieure de cet ensemble.

\section{0. - Preliminaires.}

\subsection{Polarités ([1], [4], [16], $[33], \ldots)$.}

Une application $f$ d'un ensemble ordonné $(E, \leqslant)$ dans un autre $(F,<)$ est dite isotone (resp. antitone) si pour tous $x, y$ dans $E, x \leqslant y$ implique $f(x) \leqslant f(y)$ (resp. $f(y) \leqslant f(x))$. On dira que $f$ est extensive (resp. contractante) si, pour tout $x$ dans $E, x \leqslant f(x)$ (resp. $f(x) \leqslant x)$.

$\mathrm{Si}(E, \leqslant)$ et $(F, \leqslant)$ sont des treillis complets, une correspondance de GaLoIs ([4], [32]) entre $E$ et $F$ est un couple $(\Sigma, \Omega)$ où $\Sigma$ est une application antitone de $E$ dans $F, \Omega$ une application antitone de $F$ dans $E$ et les composés $\Omega \Sigma$ et $\Sigma \Omega$ sont extensifs. 
Une polarité est une application $\Delta$ de $E$ dans $F$ telle que pour toute famile $\left(x_{i}\right)_{i \in I}$ d'éléments de $E$ on ait:

$$
\Delta \bigvee_{i \in I} x_{i}=\bigwedge_{i \in I} \Delta x_{i}
$$

D'après [38], on associe à $\Delta$ une polarité duale $\Delta^{*}: F^{*} \rightarrow E$ en posant

$$
\Delta^{*} y=\bigvee\{x \in E \mid y \leqslant \Delta x\} \quad \text { pour tout } y \in Y \text {. }
$$

On a alors l'équivalence

$$
y \leqslant \Delta x \Leftrightarrow x \leqslant \Delta^{*} y
$$

et de ce fait $\Delta=\Delta^{* *}$.

Le théorème suivant montre que, dans les treillis complets, les notions de correspondance de Galois et de polarité sont équivalentes. Il découle du théorème 3 d'EvERETt ([15̆]) alors que la notion de polarité duale a été dégagée par G. PICKFRT ([38]).

Théorìme 0.2.1 (Everett, Piokert). - Pour toute polarité $\Delta$, le couple $\left(\Delta, \Delta^{*}\right)$ est une correspondance de Galois. Inversement, si $(\Sigma, \Omega)$ est une correspondance de Galois $\Sigma$ et $\Omega$ sont des polarités et l'on a $\Omega=\Sigma^{*}$.

Les points fixes de $\Delta^{*} \Delta$, notés fix $\left(\Delta^{*} \Delta\right)$, constituent une famille d'éléments de $E$ stable par infima et de ce fait un treillis complet $([4],[11], \ldots) ; \Delta^{*} \Delta$ s'interprête comme la projection de $E$ sur fix $\left(\Delta^{*} \Delta\right)$ en ce sens que, pour tout $x \in E, \Delta^{*} \Delta x$ est le plus petit élément de fix $\left(\Delta^{*} \Delta\right)$ qui majore $x ; \Delta$ et $\Delta^{*}$ induisent des isomorphismes réciproques de treillis complets entre fix $\left(\Delta^{*} \Delta\right)$ et fix $\left(\Delta \Delta^{*}\right)^{-}$où, si $X$ est un ensemble ordonné, $X^{-}$désigne le même ensemble avec l'ordre opposé. Notons encore que fix $\left(\Delta^{*} \Delta\right)=\Delta^{*}(F)$.

Etant donné deux ensembles $X$ et $Y$, le cas particulier où $(E, \leqslant)=\left(2^{X}, c\right)$ et $(F, \leqslant)=\left(2^{Y}, C\right)$ est d'un grand intérêt et on a, pour toute famille $\left(A_{i}\right)_{i \in I}$ de parties de $X$,

$$
\Delta \bigcup_{i \in I} A_{i}=\bigcap_{i \in I} \Delta A_{i}
$$

0.2. Multiapplications-tranches ([7], [30]).

Soit $X$ un ensemble et considérons le treillis complet $\mathbb{R}^{X}$ muni de l'ordre produit:

$$
f \leqslant g \Leftrightarrow \forall x, x \in X: f(x) \leqslant g(x)
$$

ainsi que le treillis complet $2^{\mathrm{R} \times X}$ muni de l'inclusion $\subset$. 
Nous identifierons toute multiapplication $M$ de $\mathbb{R}$ dans $X$ à son graphe, c'esttàdire au sous-ensemble $\{(\lambda, x) \mid x \in M \lambda\}$ de $\mathbb{R} \times X$. Pour tous $f \in \overline{\mathbb{R}}^{X}$ et $\lambda \in \mathbb{R}$ posons

$$
\mathrm{T}_{\lambda} f=\{x \in X \mid f(x) \leqslant \lambda\} \quad \text { et } \quad T_{\lambda} f=\{x \mid f(x)<\lambda\} .
$$

Nous dirons qu'une multiapplication $M$ de $\mathbb{R}$ dans $X$ est de type tranche (resp. tranche stricte) s'il existe $f \in \overline{\mathbb{R}}^{X}$ telle que, pour tout $\lambda \in \mathbb{R}, M_{\lambda}=\mathbb{T}_{\lambda} f$ (resp. $M_{\lambda}=$ $=T_{\lambda} f$ ).

Les multiapplications-tranches (resp. tranches strictes) sont les points fixes de l'application $\wedge$ (resp. $V$ ) de $2^{\mathbb{R} \times X}$ dans lui-même définie par

$$
(\wedge M) \lambda=\bigcap_{\mu>\lambda} M_{\mu} \quad\left(\operatorname{resp} .(\vee M) \lambda=\bigcup_{\mu<\lambda} M_{\mu}\right) .
$$

En fait, il est facile de voir que la correspondance

$$
2^{\mathbb{R} \times X} \underset{\Lambda}{\stackrel{V}{\sharp}}\left(2^{\mathbb{R} \times X}\right)^{-}
$$

est de Galois. On a done les formules

$$
\begin{array}{ll}
\wedge \vee \wedge=\wedge & \vee \wedge \vee=\vee \\
\wedge \bigcap_{i \in I} M_{i}=\bigcap_{i \in I} \wedge M_{i} & \vee \bigcup_{i \in I} M_{i}=\bigcup_{i \in I} \vee M_{i} .
\end{array}
$$

Les opérateurs $\wedge$ et $\vee$ étant en outre idempotents, les points fixes de $\wedge \vee$ (resp. $\vee \wedge$ ) sont encore les multiapplications-tranches (resp. tranches strictes). En particulier, toute intersection (resp. réunion) de multiapplications-tranches (resp. tranches strictes) est de type tranche (resp. tranche stricte) et $\wedge \vee M$ (resp. $\vee \wedge M$ ) représente la plus petite (resp. grande) multiapplication-tranche (resp. tranche stricte) contenant (resp. contenu dans) $M$, pour tout $M \subset \mathbb{R} \times X$.

Maintenant, à tout $M \subset \mathbb{R} \times X$, associons la fonction Inf $M$ de $X$ dans $\overline{\mathbb{R}}$ définie par

$$
\operatorname{Inf} M(x)=\inf \{\lambda \in \mathbb{R} \mid x \in M \lambda\}
$$

Inversement, à toute fonction $f$ de $X$ dans $\overline{\mathbb{R}}$ associons les multiapplications $\mathbb{T} f$ et Tf définies en (1).

On obtient ainsi une autre correspondance de Galois

$$
2^{\mathbb{R}} \times x{\underset{\mathbb{T}}{\operatorname{Inf}}}_{\underbrace{x}} x
$$

avec les formules

$$
\operatorname{Inf\circ } \mathbb{T}^{\circ}=\inf \circ T=\mathrm{id}_{\overline{\mathbb{R}}^{x}}, \quad \operatorname{T} \circ \operatorname{Inf}=\wedge V, \quad T \circ \operatorname{Inf}=V .
$$


Au niveau des points fixes, Inf et $T$ induisent donc des isomorphismes réciproques de treillis complets entre $\left(\overline{\mathbb{R}}^{X}\right)^{-}$et le treillis complet des multiapplications-tranches.

Désignons par $\mathfrak{J}(\mathbb{R}, X)$ l'ensemble des multiapplications de $\mathbb{R}$ dans $X$ qui sont isotones en tant qu'applications de $\mathbb{R}$ dans $2^{X} ; J(\mathbb{R}, X)$ est un sous-treillis complet de $2^{\mathbb{R} \times X}$ et les correspondances ci-après sont de Galois

$$
J(\mathbb{R}, X) \underset{\wedge}{\stackrel{\vee}{t}}(J(\mathbb{R}, X))^{-}
$$

avec maintenant, pour tout $M \in \mathfrak{J}(\mathbb{R}, X)$,

$$
\begin{gathered}
\wedge \vee M=\wedge M, \quad \vee \wedge M=\vee M ; \\
J(\mathbb{R}, X) \underset{\mathbb{T}}{\stackrel{\text { Inf }}{\leftrightarrows}} \overline{\mathbb{R}}^{x}
\end{gathered}
$$

avec, pout tout $M \in \mathfrak{J}(\mathrm{R}, X)$,

$$
(\mathbb{T} \operatorname{Inf}) M=\wedge M
$$

enfin, par composition de (4) et (6), nous obtenons

$$
\left(\overline{\mathbb{R}}^{X}\right)^{-} \underset{\operatorname{Inf} \hat{\wedge}=\operatorname{Inf}}{\vee \mathbf{T}=T}(J(\mathbb{R}, X))^{-} .
$$

\section{I. - Conjugaison associée à une polarité.}

Dans ce paragraphe, $X$ et $Y$ sont deux ensembles et $\Delta: 2^{X} \rightarrow 2^{Y}$ une polarité.

\section{I.1. Conjugaison des multiapplications et des fonctions.}

Définissons $\Delta: 2^{\mathbb{R} \times X} \rightarrow 2^{\mathbb{R} \times Y}$ par:

$$
(\Delta M) \lambda=\Delta(M(-\lambda))
$$

et $\left(\Delta^{*}\right)$ semblablement par rapport à $\Delta^{*}$.

Proposition I.1.1. $-\Delta$ est une polarité et $(\Delta)^{*}=\left(\Delta^{*}\right)$.

Preuve. - Si $\left\{M_{i}\right\}_{i \in I}$ est une famille de parties de $\mathbb{R} \times X$ il vient:

$$
\left(\Delta \bigcup_{i} M_{i}\right) \lambda=\Delta\left(\bigcup_{i} M_{i}(-\lambda)\right)=\bigcap_{i} \Delta M_{i}(-\lambda)=\left(\bigcap_{i} \Delta M_{i}\right) \lambda .
$$

Par ailleurs, pour tout $N \subset \mathbb{R} \times Y,(\lambda, x) \in(\Delta)^{*} N$ si et seulement si $\Delta(\lambda, x) \supset N$. Or, $(\Delta(\lambda, x)) \mu=\Delta x$ si $\mu=-\lambda, Y$ si $\mu \neq-\lambda$. Donc, $\Delta(\lambda, x) \supset N$ si et seulement si $\Delta x \supset N(-\lambda)$ ce qui équivaut à $x \in \Delta^{*} N(-\lambda)$ c'est-à-dire à $(\lambda, x) \in\left(\Delta^{*}\right) N$. 
Dans ce qui suit nous désignerons $(\Delta)^{*}=\left(\Delta^{*}\right)$ par $\Delta^{*}$.

Remarquons que, pout tout $\lambda \in \mathbb{R},\left(\Delta^{*} \Delta M\right) \lambda=\Delta^{*} \Delta(M \lambda)$. Les points fixes de $\Delta^{*} \Delta$ sont donc les multiapplications de $\mathbb{R}$ dans $X$ dont les valeurs sont fermées pour $\Delta^{*} \Delta$. Il en résulte que $\Delta * \Delta M$ est la plus petite multiapplication de $\mathbb{R}$ dans $X$ qui contient $M$ et dont les valeurs sont fermées pour $\Delta^{*} \Delta$. Par ailleurs, on sait ([7]) que si $M$ est une multiapplication-tranche, $\Delta * \Delta M$ n'est pas nécessairement de type tranche. Notons cependant les formules

$$
\Delta \vee=\wedge \Delta, \quad \Delta^{*} \vee=\wedge \Delta^{*}
$$

Elles prouvent que $\Delta$ et $\Delta^{*}$ transforment les multiapplications-tranches strictes en multiapplieations-tranches; en outre, $\Delta$ et $\Delta^{*}$ conservent les multiapplications isotones.

Observons maintenant que, d'après [11] 1.8, la composée des correspondances de Galois ei-dessous est encore une correspondance de Galois

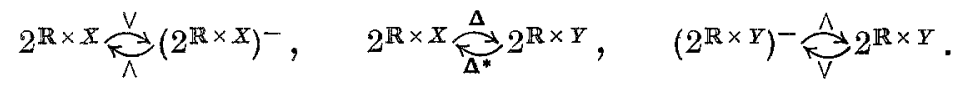

Or, d'après (10),

$$
\wedge \Delta \vee=\wedge \wedge \Delta=\wedge \Delta
$$

La conjugaison des multiapplications est alors définie par la correspondance de Galois

$$
2^{\mathbb{R} \times X} \underset{\wedge \Delta^{*}}{\stackrel{\wedge}{*}} 2^{\mathbb{R} \times Y}
$$

Les points fixes de $\wedge \Delta^{*} \wedge \Delta$ sont, par construction, les multiapplications-tranches dont les tranches sont fermées pour $\Delta^{*} \Delta$. On peut done énoncer.

THÉoRÈnE I.1.2. - Pour tout $M \subset \mathbf{R} \times X, \wedge \Delta^{*} \wedge \Delta M$ est la plus petite multiapplication-tranche parmi les multiapplications-tranches qui contiennent $M$ et dont les tranches sont fermées pour $\Delta * \Delta$.

En fait, la biconjuguée de $M \subset \mathbb{R} \times X$ s'écrit encore, d'après (10) et pout tout $\lambda \in \mathbb{R}$,

$$
\left(\wedge \Delta^{*} \Delta \vee M\right) \lambda=\bigcap_{\mu>\lambda} \Delta^{*} \Delta \bigcup_{\alpha<\mu} M \alpha
$$

Done, l'opérateur $\wedge \vee$ qui à tout $M$ associe la multiapplication-tranche, la plus petite contenant $M$ est un cas particulier de (11).

Pour obtenir la conjugaison des fonetions nous allons utiliser les opérateurs Inf et $\mathbb{T}$ et leurs propriétés résultant de $(3),(6),(8)$. 
Definimion. - Etant donné une fonction $f$ de $X$ dans $\overline{\mathbb{R}}$, la $\Delta$-conjuguée de $f$ est l'application $f^{\Delta}$ de $Y$ dans $\overline{\mathbb{R}}$ définie par

$$
f^{\Delta}=\operatorname{Inf} \wedge \Delta \mathbb{T}(f)
$$

En fait, $\Delta \mathbb{T}(f)$ étant isotone, on a d'après (8)

$$
f^{\Delta}=\operatorname{Inf} \Delta \mathbf{T}(f)
$$

soit encore, pout tout $y \in X$ et d'après (2), (9)

$$
f^{\lrcorner}(y)=\inf \left\{\lambda \mid y \in \Delta\left(\mathbb{T}_{-\lambda} f\right)\right\} .
$$

De même, pour $g \in \overline{\mathbb{R}}^{Y}$, nous aurons

$$
g^{\Delta^{*}}=\operatorname{Inf} \Delta^{*} \mathrm{~T}(g)
$$

On obtient ainsi une nouvelle correspondance de Galois

$$
\left(\overline{\mathbb{R}}^{X}\right)^{-} \underset{\operatorname{Inf} \Delta^{* T}}{\stackrel{\operatorname{Inf} \Delta T}{\Delta}}\left(\overline{\mathbb{R}}^{Y}\right)^{-}
$$

qui résume la conjugaison des fonctions.

Liming I.1.3. - La formule suivante est vérifiée:

$$
\mathbb{T} \operatorname{Inf} \Delta \mathbb{T}=\Delta \mathbb{T}
$$

Preuve. - D'après (7), (10), (8) il vient respectivement

$$
\mathbb{T} \operatorname{Inf} \Delta \mathbb{T}=\wedge \Delta \mathbb{T}=\Delta \vee \mathbb{T}=\Delta T .
$$

Proposition I.1.4. - L'opérateur de biconjugaison des fonctions de $X$ dans $\overline{\mathbb{R}}$ s'écrit $\operatorname{Inf} \Delta * \Delta \mathbb{T}$.

Prevpe. - D'après (13) cet opérateur est donné par Inf $\Delta^{*} \mathbb{T} \operatorname{Inf} \Delta T$; il suffit alors d'appliquer le lemme I.1.3.

Les fonctions $f \in \overline{\mathbb{R}}^{X}$ qui coïncident avec leur biconjuguée sont, par construction, celles dont les tranches sont fermées pour l'opérateur $\Delta^{*} \Delta$; on peut done énoncer:

THÉOR亡̀Me I.1.5. - Pour toute fonction $f$ de $X$ dans $\overline{\mathbb{R}}, f^{\Delta \Delta^{*}}$ est la plus grande fonction qui minore $f$ et dont les tranehes sont fermées pour $\Delta^{*} \Delta$. 
Le théorème suivant donne explicitement les tranches des fonctions conjuguées.

THÉORظ̀me I.1.6. - Pour toute fonction $f \in \overline{\mathbb{R}}^{x}$ et tout réel $\lambda$ on a les formules
i) $\mathbb{T}_{\lambda} f^{\Delta}=\Delta\left(T_{-\lambda} f\right)$
ii) $\mathbb{T}_{\lambda} f^{\Delta \Delta^{*}}=\bigcap_{\mu>\lambda} \Delta^{*} \Delta \mathbb{T}_{\mu} f$
iii) $\mathbb{T}_{\lambda} f^{\Delta \Delta^{*}}=\bigcap_{\mu>\lambda} \Delta^{*} \Delta T_{\mu} f$.

PREuve. - i) S'écrit en fait $\mathbb{T} \operatorname{Inf} \Delta \mathbb{T}=\Delta T$ et on applique le lemme I.1.3.

ii) D'après la proposition I.1.4, la formule à démontrer est iei T Inf $\Delta^{*} \Delta \mathrm{T}=$ $=\wedge \Delta * \Delta \mathrm{T}$ ce qui est immédiat d'après (7).

iii) D'après (8), (10), (5), il vient

$$
\wedge \Delta^{*} \Delta T=\wedge \Delta * \Delta \vee \mathbb{T}=\wedge \Delta^{*} \wedge \Delta \mathbb{T}=\Delta^{*} \vee \wedge \Delta \mathbb{T}=\Delta^{*} \vee \Delta T=\wedge \Delta^{*} \Delta \mathbb{T}
$$

ce qui, avec ii), permet de conclure.

On peut en déduire des formules explicites pour les fonctions conjuguées.

CorollarRe I.1.7. - Pour toute fonction $f \in \overline{\mathbb{R}}^{X}$ et tout $x \in X$ on a:
i) $f^{\Delta}(x)=\inf \left\{\lambda \mid x \in \Delta\left(T_{-\lambda} f\right)\right\}$;
ii) $f^{\Delta \Delta^{*}}(x)=\inf \left\{\lambda \mid x \in \Delta^{*} \Delta\left(\mathbb{T}_{\lambda} f\right)\right\}$;
iii) $f^{\Delta \Delta^{*}}(x)=\inf \left\{\lambda \mid x \in \Delta^{*} \Delta\left(T_{\lambda} f\right)\right\}$.

Preuve. - i) Résulte immédiatement du théorème I.1.6. i); ii) et iii) s'obtiennent à partir du théorème T.1.5. ii), iii) en observant que $\Delta^{*} \Delta \mathbf{T}(f)$ et $\Delta^{*} \Delta T(f)$ sont isotones et en appliquant (8).

Remarques. - 1) Puisque ${ }^{{ }^{\top}} \mathbb{T}_{+\infty} f=X$ et $\mathbb{T}_{-\infty} f=\bigcap_{\lambda \in \mathbb{R}}{ }^{\top} \mathbb{T}_{\lambda} f$, nous déduisons du théorème I.1.5 que $f=f^{\Delta d^{*}}$ si et seulement si, pour tout $\lambda \in \overline{\mathbb{R}}, \mathbb{T}_{\lambda} f=\Delta^{*} \Delta \mathbb{T}_{\lambda} f$.

2) D'après (13), l'opérateur de conjugaison $f \mapsto f^{\Delta}$ est une polarité entre les treillis complets $\left(\overline{\mathbb{R}}^{X}\right)^{-}$et $\left(\overline{\mathbb{R}}^{Y}\right)^{-}$; cela se traduit par la formule

$$
\left(\inf _{i \in I} f_{i}\right)^{A}=\sup _{i \in I} f_{i}^{A}
$$

valable pour toute famille $\left(f_{i}\right)_{i \in I}$ de fonctions de $X$ dans $\overline{\mathrm{R}}$. 
3) L'opérateur $g \mapsto g^{\Delta^{*}}$ est la polarité duale de la précédente; on a donc, pour tout $g \in \overline{\mathbb{R}}^{Y}$

$$
g^{\mathbf{d}^{*}}=\inf \left\{f \in \overline{\mathbb{R}}^{x} \mid g \geqslant f^{\Delta}\right\} .
$$

En outre, les fonctions dont les tranches sont fermées pour $4 * \Delta$ sont celles $d u$ type $g^{\Delta^{*}}$ lorsque $g$ parcourt $\overline{\mathbb{R}}^{T}$.

I.2. Comparaison avec la conjugaison de Fenchel génératisée.

Considérons comme dans [30], les deux prolongements de l'addition de $\mathbb{R} \times \mathbb{R}$ à $\overline{\mathbb{R}} \times \overline{\mathbb{R}}$ ainsi définis:

\begin{tabular}{l|l|l|}
+ & $+\infty$ & $-\infty$ \\
\hline$+\infty$ & $+\infty$ & $+\infty$ \\
\hline$-\infty$ & $+\infty$ & $-\infty$ \\
\hline
\end{tabular}

\begin{tabular}{c|c|c|}
+ & $+\infty$ & $-\infty$ \\
\hline$+\infty$ & $+\infty$ & $-\infty$ \\
\hline$-\infty$ & $-\infty$ & $-\infty$ \\
\hline
\end{tabular}

Rappelons ([3], [14], [17], [23], [29], [30],...) qu'étant donné deux ensembles $X, Y$ et une fonction de couplage $\gamma: X \times Y \rightarrow \overline{\mathbb{R}}$ on appelle conjuguée d'une fonetion $f \in \overline{\mathbb{R}}^{X}$ (resp. $g \in \overline{\mathbb{R}}^{Y}$ ) la fonction $f^{\gamma} \in \overline{\mathbb{R}}^{Y}$ (resp. $g^{\gamma} \in \overline{\mathbb{R}}^{X}$ ) définie par:

$$
f^{\gamma}-(y)=\sup _{x \in X}(\gamma(x, y)+(-f(x))) \quad\left[\operatorname{resp} . g^{\gamma}(x)=\sup _{y \in Y}(\gamma(x, y)+(-g(y)))\right] .
$$

On a alors ([30])

$$
f^{\gamma \gamma}(x)=\sup \{\gamma(\cdot, y)+r \mid(y, r) \in Y \times \mathbb{R}, \gamma(\cdot, y)+r \leqslant f\} .
$$

Nous allons voir que cette généralisation de la conjugaison de Fenchel nous permet de retrouver la conjugaison définie par (13). Pour cela considérons la fonction de couplage

$$
\delta: X \times Y \rightarrow \overline{\mathbb{R}}, \quad \delta(x, y)=-\infty \quad \text { si } y \in \Delta x, \quad 0 \quad \text { si } y \notin \Delta x .
$$

D'après (14) on obtient ainsi pour tous $f \in \overline{\mathbb{R}}^{x}, g \in \overline{\mathbb{R}}^{T},(a, b) \in X \times Y$ :

$$
f^{\delta}(b)=-\inf _{x \notin \Delta^{*} b} f(x), \quad g^{\delta}(a)=-\inf _{y \notin \Delta_{a}} g(y) .
$$

THÉoRÈme I.2.1. - Pour tous $f \in \overline{\mathbb{R}}^{X}$ et $g \in \overline{\mathbb{R}}^{Y}, f^{\delta}=f^{4}, g^{\delta}=g^{\Delta^{*}}$.

Preuve. - Quitte à échanger $\Delta$ et $\Delta^{*}$, il suffit de montrer que $f^{\delta}=f^{\Delta}$ et pour cela que, pour tout $\lambda \in \mathbb{R}, \mathbb{T}_{\lambda} f^{\delta}=\mathbb{T}_{\lambda} f^{\Delta}$. Or,

$$
\begin{aligned}
\mathbb{T}_{\lambda} f^{\delta}=\left\{y \in Y \mid x \notin \Delta^{*} y \Rightarrow f(x) \geqslant-\lambda\right\} & =\{y \in Y \mid f(x)<-\lambda \Rightarrow y \in \Delta x\}= \\
& =\bigcap_{x \in T_{-\lambda} f} \Delta x=\Delta T_{-\lambda} f=\mathbb{T}_{\lambda} f^{\Delta} . \quad \text { (Th. I.1.6. i) }
\end{aligned}
$$

19 - Annali di Matematica 
COROLIATRE I.2.2. - Pour tout $f \in \overline{\mathbb{R}}^{x}$ il y a équivalence entre:

i) Les tranches de $f$ sont fermées pour $\Delta^{*} \Delta$.

ii) Il existe une famille $\left\{y_{k}\right\}_{k \in \Gamma}$ d'éléments de $Y$ telle que $f=\sup _{k \in \bar{K}} f_{k}$ ò̀ chaque $f_{k}$ est une fonotion qui vaut $-\infty$ sur $\Delta^{*} y_{k}$ et est constante et finie ailleurs.

Preuvz. - i) équivaut à $f=f^{\delta \delta}$ soit, d'après (15), à l'existence d'une famille $\left(y_{k}, r_{k}\right)_{k \in K}$ d'éléments de $Y \times \mathbb{R}$ telle que $f=\sup _{k}\left\{\delta\left(\cdot, y_{k}\right)+r_{k}\right\}$, ce qui permet de conclure.

Maintenant, nous allons voir que tonte conjugaison au sens de Fenchel généralisé est entièrement déterminée par la conjugaison relative à une polarité bien choisie. Pour ce faire, le lemme suivant étudie la régularisée par tranches d'une fonction indicatrice. Notons que l'hypothèse $\Delta^{*} Y=\emptyset$ équivaut à $\Delta^{*} \Delta \emptyset=\emptyset$ ou encore à $\Delta x \neq Y$ pour tout $x \in X$.

Notations. - Etant donné un ensemble $E$, la fonction indicatrice d'une partie $A$. de $E$ et le complémentaire de $A$ dans $E$ seront respectivement notés $\psi_{A}$ et $A^{c}$. Si $f \in \overline{\mathbb{R}}^{E}$, on pose Epi $f=\{(x, \lambda) \in E \times \mathbb{R} \mid f(x) \leqslant \lambda\}$.

LeMñE I.2.3. - Pout toure partie A de $X$ l'on a

i) $\left(\psi_{A}\right)^{A}=-\psi_{(A A)^{c}}$;

ii) $\operatorname{sic} \Delta^{*} Y=\emptyset,\left(\psi_{A}\right)^{\Delta \Delta^{*}}=\psi_{A^{*} \Delta A}$.

Preuve. $-\left(\psi_{A}\right)^{A}(y)=-\inf _{x \neq A^{*} y} \psi_{A}(x)=0$ si $\left(\Delta^{*} y\right)^{c} \cap A \neq \emptyset,-\infty$ sinon. Or, $\left(A^{*} y\right)^{c} \cap$ $\cap A=\emptyset \Leftrightarrow A \subset \Delta^{*} y \Leftrightarrow y \in \Delta A ;$ a'où i).


$x \in \Delta^{*} \Delta A$.

THEOREME I.2.4. - Soit $\gamma: X \times \bar{Y} \rightarrow \overline{\mathbb{R}}$ une fonction de couplage et $\Gamma$ l'application de $2^{X \times \mathbb{R}}$ dans $2^{\bar{Y} \times \mathbb{R}}$ définie, pour tout $D \subset X \times \mathbb{R}$, par

$$
\Gamma D=\{(y, s) \in Y \times \mathbb{R} \mid(x, r) \in D \Rightarrow \gamma(x, y)-r-s \leqslant 0\} .
$$

$\Gamma$ est une polarité et, pour toute fonction $f \in \overline{\mathbb{R}}^{x}$,

i) Epi $f^{\gamma}=\Gamma($ Epi $f)$; Epi $f^{\gamma \gamma}=\Gamma^{*} \Gamma($ Epi $f)$;

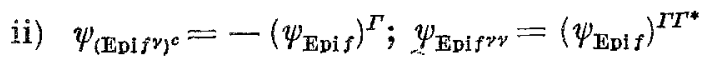

avec, pour la dernière formule, l'hypothèse: $\forall x \in X, \exists y \in Y \mid \gamma(x, y) \neq-\infty$. 
Preuve. - Il est facile de voir que $\Gamma$ est une polarité. En outre, d'après le lemme I.2.3 et par symétrie, il suffit de montrer la première formule de i). Or, pour tout $(y, s) \in Y \times \mathbb{R}$, on a les équivalences:

$$
\begin{aligned}
f^{\gamma}(y) \leqslant s & \Leftrightarrow \gamma(\cdot, y)+(-f) \leqslant s \Leftrightarrow \gamma(\cdot, y)-s \leqslant f \Leftrightarrow \\
& \Leftrightarrow(f(x) \leqslant r \Rightarrow \gamma(x, y)-s \leqslant r) \Leftrightarrow \\
& \Leftrightarrow(y, s) \in \Gamma(\text { Epi } f) . \quad \square
\end{aligned}
$$

I.3. Quasidifférentiabilité relative à une polarité.

Observons que, d'après Th. I.2.1, on a la formule fondamentale

$$
f^{\Delta \Delta^{*}}(a)=\sup _{y \notin \Delta a} \inf _{x \notin \Delta^{*} v} f(x)
$$

Définition. - Nous disons que $b \in Y$ est un $\Delta$-quasigradient de $f$ en $a \in X$, et on note $b \in \partial^{\Delta} f(a)$, si $f(a)=f^{\Delta \Delta^{*}}(a)$ et si $b$ réalise le sup dans la formule (18).

Autrement dit: $b \in \partial^{\Delta} f(a) \Leftrightarrow b \notin \Delta a$ et $f(a)=-f^{\Delta}(b)$.

En fait, $b \in \partial^{A} f(a)$ si et seulement si $a$ est solution optimale du problème aminimiser $f(x)$ sous la contrainte $x \notin \Delta^{*} b$ ).

De même, pour $g \in \overline{\mathbb{R}}^{x}, a \in \hat{\partial}^{\Delta^{*}} g(b) \Leftrightarrow a \notin \Delta^{*} b$ et $g(b)=-g^{\Delta^{*}}(a)$.

Proposition I.3.1. - Les propriétés suivantes sont vérifiées:

i) $b \in \partial^{\Delta} f(a) \Leftrightarrow b \notin \Delta a$ et $\forall x \in X, f(x)+(-f(a)) \geqslant \delta(x, b)+(-\delta(a, b))$.

ii) $f(a)=f^{\Delta \Delta^{*}}(a)$ implique $\partial^{\Delta} f(a)=\partial^{\Delta} f^{\Delta \Delta^{*}}(a)$.

iii) Si $f(a)=f^{\Delta \Delta^{*}}(a)$ alors $b \in \partial^{\Delta} f(a) \Leftrightarrow a \in \partial^{\Delta^{*}} f^{\Delta}(b)$.

iv) Si $\Delta^{*} b=\emptyset$ alors $b \in \partial^{4} f(a) \Leftrightarrow f(a)=\inf f$.

Preuve. - i): Résulte de (16) et du fait que $f(x)+(-f(a)) \geqslant 0 \Leftrightarrow f(x) \geqslant f(a)$.

ii): $b \in \partial^{\Delta} f^{\Delta \Delta^{*}}(a)$ signifie que $b \notin \Delta a$ et $f^{\Delta \Delta^{*}}(a)=-f^{\Delta \Delta^{*} \Delta}(b)$. Or, d'après 13 , $f^{\Delta \Delta^{*} \Delta}=f^{\Delta}$ ce qui permet de conclure.

iii): Si $f(a)=f^{\Delta \Delta^{*}}(a)$ on a les équivalences

$$
b \in \partial^{\Delta} f(a) \Leftrightarrow\left(b \notin \Delta a \quad \text { et } \quad f^{\Delta}(b)=-f^{\Delta \Delta^{*}}(a)\right) \Leftrightarrow a \in \partial^{\Delta^{*}} f^{\Delta}(b) .
$$

iv): Il suffit de noter que $\Delta^{*} b==\emptyset$ implique $f^{\Delta}(b)=-\inf f$.

Le théorème suivant caractérise le $\Delta$-quasidifférentiel $\partial^{\Delta} f(a)$ par une formule qui permettra, dans les exemples qui suivent, de donner la structure de celui-ci. 
THÉORł̇ME I.3.2. - Les égalités suivantes ont toujours lieu:
i) $\partial^{\Delta} f(a)=\left\{b \in(\Delta a)^{c} \mid \forall \lambda<f(a): \mathbb{T}_{\lambda} f \subset \Delta^{*} b\right\}$;
ii) $\partial^{\Delta} f(a)=(\Delta a)^{c} \cap \Delta T_{f(a)} f$;
iii) $\partial^{4} f(a)=\left\{b \in(\Delta a)^{c} \mid \forall \lambda<f(a): T_{\lambda} f \subset \Delta^{*} b\right\}$.

Preuve. - i): Soit $b \notin \Delta a$. Il suffit de montrer que $f(a)=-f^{\Delta}(b)$ équivaut au fait que, pour tout $\lambda<f(a), \mathbb{T}_{\lambda} f \subset \Delta^{*} b$. Vu que $b \notin \Delta a$, on a ici $f(a) \geqslant-f^{\Delta}(b)$. Maintenant, si $f(a) \leqslant-f^{\Delta}(b)$ et si $\lambda<f(a), x \notin \Delta^{*} b$ implique $f(x)>\lambda$ et, de ce fait, $\mathbb{I}_{\lambda} f \subset$ $c \Delta^{*} b$. Réeiproquement, soit $\lambda<f(a)$ et $x \notin \Delta^{*} b$; on a alors $f(x)>\lambda$, d'où $\inf _{x \notin A^{*} b} f(x) \geqslant \lambda$ et, en passant au sup sur les $\lambda, \inf _{x \notin \Delta^{*}} f(x) \geqslant f(a)$.

ii): D'après i),

$$
\partial^{\Delta} f(a)=(\Delta a)^{c} \cap\left\{b \mid \cup \mathbb{T}_{\lambda<f(a)} \mathbb{T}_{\lambda} f \subset \Delta^{*} b\right\}=(\Delta a)^{c} \cap\left\{b \mid T_{f(a)} \subset \Delta^{*} b\right\}=(\Delta a)^{e} \cap \Delta T_{f(a)} f
$$

iii): Résulte de i) et du fait que $\bigcup_{\lambda<f(a)} \mathbb{T}_{\lambda} f=\bigcup_{\lambda<f(a)} T_{\lambda} f$.

Pour ce qui est de la propriété $f(a)=f^{\Delta \Delta^{*}}(a)$, on a le théorème suivant, dans lequel on remarquera que $f$ est $\Delta$-quasidifférentiable en a si et seulement si le $y$ ne dépend pas du 2. Dans un contexte différent, un résultat analogue est donné dans [28] en utilisant la conjugaison au sens de LINDBERG [25].

THÉORłiver I.3.3. - Les propriétés suivantes sont équivalentes:

i) $f(a)=f^{\Delta \Delta^{*}}(a)$;

ii) $\forall \lambda<f(a), \exists y \in X \mid a \notin \Delta^{*} y$ et $\mathbb{T}_{\lambda} f \subset \Delta^{*} y$;

iii) $\forall \lambda<f(a), \exists y \in Y \mid a \notin \Delta^{*} y$ et $T_{\lambda} f \subset \Delta^{*} y$.

Preupte. - i) $\Rightarrow$ ii): Soit $\lambda<f(a)$. D'après (18) il existe $y \notin \Delta a$ tel que $\underset{x \neq \Delta^{*} y}{\operatorname{not}} f(x)>\lambda$. $x \notin \Delta^{*} y$ implique done $f(x)>\lambda$ et on a bien $\mathbb{T}_{\lambda} f \subset \Delta^{*} y$.

ii) $\Rightarrow$ iii) est évident; montrons que iii) $\Rightarrow$ i). Soit $\lambda<f(a)$; il existe $y$ tel que $a \notin \Delta^{*} y$ et $y \in \Delta T_{A} f$. D'après (18), $f^{\Delta A^{*}}(a) \geqslant-f^{\Delta}(y)$ et d'après Cor. I.1.7. i), $-f^{\Delta}(y)=$ $=-\inf \left\{\mu \mid y \in \Delta T_{-\mu} f\right\}=\sup \left\{\mu \mid y \in \Delta T_{\mu} f\right\}$. On a alors $f^{\Delta \Delta^{*}}(a) \geqslant-f^{\Delta}(y) \geqslant \lambda$ ce qui permet de conclure.

REMaRque. - Les propriétés ii) et iii) expriment que l'on peut séparer une tranche de $f$ d'un point par un ensemble du type $\Delta^{*} y$.

Dans la proposition suivante on étudie la $\Delta$-quasidifférentiabilité d'une fonction indicatrice. 
Proposition I.3.4. - Pour toute partie $A$ de $X$ on $a$ :

i) $\partial^{\Delta} \psi_{A}(a)=(\Delta a)^{c}$ si $a \in A$.

ii) $\partial^{\Delta} \psi_{A}(a) \neq \emptyset$ si $A=\Delta^{*} \Delta A$ et $a \notin A$.

Preuve. - i): On a ici $\left\{x \mid \psi_{A}(x)<\psi_{A}(a)\right\}=\emptyset$ et, d'après Th. I.3.2 ii), $\partial^{\Delta} \psi_{\Delta}(a)=$ $=(\Delta a)^{c}$.

ii): Maintenant $\left\{x \mid \psi_{A}(x)<\psi_{A}(a)\right\}=A$; en outre $a \notin \Delta^{*} \Delta A$ équivaut à $\Delta a \not \supset \Delta A$ de sorte que $\partial^{\Delta} \psi_{A}(a)=(\Delta a)^{e} \cap \Delta A \neq \emptyset$.

\section{II. - Exemples.}

Avant de remarquer, au § III, que nombre de conjugaisons introduites par divers auteurs en quasiconvexité sont en fait des conjugaisons relatives à des polarités, nous montrons que la conjugaison par tranches que nous venons de définir dépasse largement le cadre de la quasiconvexité en étudiant des exemples de nature ensembliste, algébrique et topologique. L'obtention d'une conjugaison exacte pour les fonctions s.c.i., quasiconvexes, monotones, pour les $V$-morphismes de treillis complet etc. constitue le résultat principal de ce paragraphe.

\section{II.1. Conjugaison associée à un opérateur de fermeture.}

Soit $X$ un ensemble, $A \mapsto \vec{A}$ un opérateur de fermeture sur $2^{x}$ et $\mathfrak{B}$ une base intersectionnelle de fermés (pour tout $A \subset Y, \bar{A}$ est l'intersection des éléments de $\mathfrak{B}$ qui contiennent $A$ ). A cet ojérateur on associe la polarité $\Delta: 2^{x} \rightarrow 2^{\mathfrak{B}}$ définie par:

$$
\Delta A=\{B \in \mathscr{3} \mid A \subset B\} \quad \text { (voir aussi }[24],[15],[31])
$$

Il vient alors, pour tous $A \subset X, \mathcal{A} \subset \mathscr{B}: \Delta^{*} \mathcal{A}=\bigcap_{B \in \mathcal{A}} B, \Delta^{*} \Delta A=\bar{A}$.

Il en résulte que tout opérateur de fermeture sur $2^{x}$ s'écrit sous la forme $\Delta^{*} \Delta$ où $\Delta$ est une polarité. Une telle décomposition n'est pas unique car chaque paramétrisation d'une base de fermés fournit une polarité différente. Le théorème suivant résulte immédiatement des résultats obtenus au $\$ \mathrm{~T}$.

THÉoRÈme II.1.1. - Etant donné un ensemble $X$, un opérateur de fermeture sur $2^{X}$ défini par $A \mapsto \bar{A}$ et la polarité définie en (19) on a, pour tous $f \in \overline{\mathbb{R}}^{X}, a \in X, A \in \Re$.

i) $f^{\Delta}(A)=-\inf _{x \notin A} f(x)=\inf \left\{\lambda \mid \mathbb{T}_{-\lambda} f \subset A\right\}=\inf \left\{\lambda \mid T_{-\lambda} f \subset A\right\}$

ii) $f^{\Delta \Delta^{*}}(a)=\sup _{a \neq \bar{\ddagger}} \inf _{x \notin B} f(x)=\inf \left\{\lambda \mid a \in \overline{\mathrm{T}_{\lambda} f}\right\}=\inf \left\{\lambda \mid a \in \overline{T_{\lambda} f}\right\} ;$

iii) $B \in \partial^{4} f(a) \Leftrightarrow a$ est solution optimale du problème $\inf _{x \neq \mathcal{B}} f(x)$. 
iv) $f(a)=f^{\Delta \Delta^{*}}(a) \Leftrightarrow \forall \lambda<f(a), \exists B \in \mathfrak{B} / a \notin B$ et $B \supset T_{\lambda} f$,

$$
\Leftrightarrow \quad \text { " " } \quad B \supset \mathbb{T}_{\lambda} f \text {; }
$$

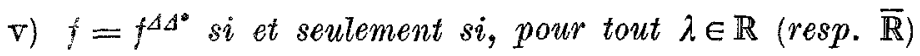

$\overline{\mathbb{T}_{\lambda} f}=\mathbb{T}_{\lambda} f$ ou encore si et seulement si il exisle une famille $\left\{B_{k}\right\}_{k \in K}$ d'élément de $\mathfrak{B}$ ei une famille $\left\{\alpha_{k}\right\}_{k \in K}$ de réels tels que, pour tout $x \in X, f(x)=\sup _{x \notin B_{k}} \alpha_{k}$.

Nous avons done caractérisé par biconjugaison exacte et symétrique les fonctions réelles dont les tranches sont fermées pour un opérateur donné. Voici trois exemples:

a) Fermeture topologique. - $X$ est un espace topologique et $\mathcal{B}$ une base de fermés. Pour tout $a \in X$, la famille $\left\{B^{c} \mid B \in \mathfrak{B}\right.$ et $\left.a \notin B\right\}$ est une base de voisinages de $a$, de sorte que, d'après Th. II.1.1. (iii), et pour tout $f \in \overline{\mathbb{R}}^{x}$ :

$$
f^{\Delta \Delta^{*}}(a)=\sup _{a \in B^{*}} \inf _{x \in B^{*}} f(x)=\liminf _{x \rightarrow a} f(x)
$$

$f^{\Delta \Delta^{*}}$ n'est autre que la régularisée s.c.i. de $f$. Fn outre,

Proposition II.1.2. - $f$ est $\Delta$-quasidifférentiable en a si et seulement si $f$ atteint en a un minimum local.

Preuve, $-f(a)$ est minimum local de $f$ si et seulement si il existe $B$ dans $B$ tel que $a \in B^{c}$ et $f(a)=\inf _{x \in B^{c}} f(x)$ soit encore $B \notin \Delta a$ et $f(a)=-f^{\Delta}(B)$.

b) Enveloppe convexe. - Soit $X$ un $\mathbb{R}$-espace vectoriel et $\mathcal{C}$ une base intersectionnelle des parties convexes de $X . f^{\Delta \Delta^{*}}$ est ici la régularisée quasiconvexe de $f$ et nous aurons, en désignant par co $A$ l'enveloppe convexe de $A \subset X$ et en appliquant Th. II.1.1. ii):

$$
f^{\Delta \Delta^{*}}(a)=\inf \left\{\lambda \mid a \in \operatorname{co} \mathbb{T}_{\lambda} f\right\}=\inf \left\{\lambda \mid a \in \operatorname{co} T_{\lambda} f\right\}
$$

On retrouve ainsi le procédé utilisé par CRouztix ([7]) pour obtenir la régularisée quasiconvexe. Notons encore que, d'après Th. II.1.1 iv), il est possible de définir la notion de fonction quasiconvexe en a par la propriété

$$
\forall \lambda<f(\alpha), \exists \Omega^{c} \in \mathcal{C} \mid a \in \Omega \quad \text { et } \quad(x \in \Omega \Rightarrow f(x)>\lambda) .
$$

Remarquons que (20) s'obtient à partir de la propriété $f$ s.c.i. en $a$ en remplaçant les ouverts par les complémentaires des convexes et que $f$ est quasiconvexe si et seulement si (20) est vraie en tout point $a \in X$. D'après Th. II.1.1 v), une fonction quasiconvexe apparait comme enveloppe supérieure de fonctions prenant la valeur $-\infty$ sur un convexe et une valeur constante et finie ailleurs, on retrouve ainsi une propriété mise en évidence par DURIER ([12]). 
THÉonÈME II.1.3. - Si $\mathrm{C}$ est la famille de toutes les parties convexes de $X$ et si $\Delta$ est la polarité définie en (19), toute fonction quasiconvexe $f: X \rightarrow \overline{\mathbb{R}}$ est $\Delta$-quasidifférentiable en tout point.

Preuve. - Soit $a \in X$ et $B=\{x \in X \mid f(x)<f(a)\}$. B est une partie convexe qui ne contient pas $a$ et l'on a, pour tout $\lambda<f(a), B \supset \mathbb{T}_{\lambda} f$. Le théorème I.4.2 i) permet alors de conclure.

c) F'ermeture décroissante. $-X$ est un ensemble ordonné par $\leqslant$ et on considère la polarité $\Delta: 2^{x} \rightarrow 2^{x}$ définie, pour tout $A \subset X$, par

$$
\Delta A=d(A)^{c} \quad \text { où } \quad d(A):=\{x \in X|\exists a \in A| x \leqslant a\} .
$$

On a alors, pour toute partie $B \subset X$,

$$
\Delta^{*} B=i(B)^{\circ} \quad \text { où } \quad i(B):=\{x \in X|\exists b \in B| b \leqslant x\}
$$

ainsi que

$$
\Delta^{*} \Delta A=\left(i\left(d(A)^{c}\right)\right)^{c}=d(A)
$$

d est l'opérateur d'enveloppe décroissante et les fonctions dont les tranches sont fermées pour $d$ sont les fonctions croissantes de $X$ dans $\overline{\mathbb{R}}$ ([5]). Pour tout $f \in \overline{\mathbb{R}}^{x}$, $f^{\Delta \Delta^{*}}$ est la régularisée croissante de $f$ et l'on a

$$
f^{\Delta \Delta^{*}}(a)=\sup _{y \leqslant a} \inf _{x \geqslant y} f(x)=\inf _{x \geqslant a} f(x)
$$

ce qui prouve que l'on a toujours $a \in \partial^{\Delta} f^{\Delta \Delta^{*}}(a)$.

Symmétriquement

$$
\Delta \Delta^{*} B=\left(d\left(i(B)^{c}\right)\right)=i(B)
$$

de sorte que $f^{\Delta^{* \Delta}}$ est la régularisée décroissante de $f$.

Dans le cas où $X$ est l'ensemble des parties d'un ensemble $E$, ordonné par l'inclusion, considérons la polarité $\Gamma: 2^{x} \rightarrow 2^{x}$ définie pour tout $\mathfrak{A} \subset 2^{x}$ par

$$
\Gamma \mathfrak{U}=\mathfrak{Q}^{\sharp f}:=\{B \subset E \mid \forall \mathcal{A} \in \mathfrak{Q}: A \cap B \neq \emptyset\} \text {. }
$$

$\Gamma \mathfrak{A}$ est la grille de $\mathfrak{A}([6],[11])$. On a ici $\Gamma=\Gamma^{*}$ et

$$
\Gamma^{2}(\mathfrak{U})=\{A \subset E|\exists B \in \mathfrak{A}| B \subset A\}=i(\mathfrak{Q}) .
$$


Il en résulte que, pour tout $F: 2^{E} \rightarrow \overline{\mathbb{R}}, F^{\Gamma \Gamma}$ est la régularisée décroissante de $F$ et que pour tout $A \subset E$,

$$
\left.F^{T T}(A)=\sup _{B \cap A=\emptyset} \inf _{D \cap B=\emptyset} F(D) \quad \text { (comparer avec }(21)\right)
$$

On vérifie quo $A^{a} \in \partial^{A} f^{r F}(A)$ et on obtient bien

$$
F^{T T}(A)=\inf _{D \subset A} F(D)
$$

II.2. Caracierisation des $\vee$-morphismes de treillis complets a valeurs dans $\overline{\mathbb{R}}$.

Soit $(\mathscr{E}, \leqslant)$ un treillis complet et considérons la polarité $\Delta$ de $2^{E}$ dans lui même définie par $\Delta A=\{y \in E \mid \sup A \leqslant y\}$ pour tout $A \subset E$. On a alors, pour tout $(A, B) \in$ $\in E \times E$,

$$
\Delta^{*} B=\{x \in \mathbb{E} \mid x \leqslant \inf B\} ; \quad \Delta * \Delta A=\{x \in E \mid x \leqslant \sup A\} .
$$

THéoR̀̀ise II.2.1. - Pour tout $f \in \overline{\mathbb{R}}^{E}$, il y a équivalenee entre:

i) $f=f^{\Delta \Delta^{*}}\left(\right.$ resp. $\left.f=f^{\Delta^{*} \Delta}\right)$.

ii) Pour toute famille $\left\{x_{k}\right\}_{k \in K}$ d'éléments de $E, f\left(\sup _{k} x_{k}\right)=\sup _{k} f\left(x_{k}\right)($ resp. $\left.f\left(\inf _{k} x_{k}\right)=\sup _{k} f\left(x_{k}\right)\right)$.

Preuve. - i) $\Rightarrow$ ii). Montrons que $f$ est croissante. Si $x \leqslant t$ on a $x \leqslant$ sup $\mathbb{T}_{f(t)} f$. Or, $\mathbb{T}_{f(t)} f$ est fermé pour $\Delta^{*} A$; on a donc, d'après $(22), x \in \mathbb{T}_{f(t)} f$ c'est-à-dire $f(x) \leqslant$ $\leqslant f(t) . \quad f$ est done croissante et l'on a $\sup _{k} f\left(x_{k}\right) \leqslant f\left(\sup _{k} x_{k}\right)$. En outre, $\sup _{k} x_{k} \leqslant$ $\leqslant \sup \mathbb{T}_{\substack{\text { supf } f\left(x_{k}\right) \\ k}} f$ implique $\sup _{k} x_{l k} \in \mathbb{T}_{\substack{\text { sup } f^{\prime}\left(x_{k}\right) \\ f}} f$ et l'on a donc $f\left(\sup _{k} x_{k}\right) \leqslant \sup _{k} f\left(x_{k}\right)$.

ii) $\Rightarrow$ i). Il s'agit de montrer que, pour tout $\lambda \in \mathbb{R}, x \leqslant \sup \mathbb{T}_{\lambda} f$ implique $f(x) \leqslant \lambda$. Or, en notant que $f$ est nécessairement croissante, $x \leqslant \sup \mathbb{T}_{\lambda} f$ implique $f(x) \leqslant$ $\leqslant f\left(\sup \mathbb{T}_{\lambda} f\right)=\sup _{x(x) \leqslant \lambda} f(x) \leqslant \lambda$. La démonstration s'achève en notant que si l'on change l'ordre de $E$ en son opposé $\Delta$ et $\Delta^{*}$ permutent.

\section{III. - Conjugaison quasiconvexes.}

Jusqu'à nouvel ordre, $X$ et $Y$ seront des R-espaces localement convexes séparés mis en dualité séparante par la forme bilinéaire $\langle\rangle:, X \times Y \rightarrow \mathbb{R}$.

III.1. Fonotions quasi «evenly» convexes propres.

En accord aveo [18] nous qualifierons d'" evenly " convexe toute intersection de demi-espaces ouverts de $X$ (ou de $Y$ ). Par exemple, tout convexe fermé est "evenly" 
convexe. Une fonction $f \in \overline{\mathbb{R}}^{x}$ sera dite quasi "evenly" convexe si ses tranches sont "evenly" convexes. D'après la terminologie de [34], nous dirons qu'une fonction homogène quasi "evenly" convexe est propre si $f(0)=\sup f$. (Homogène signifiant $f(\lambda x)=f(x), \forall \lambda>0, \forall x \in X$.) Soit alors la polarité $\Delta: 2^{X} \rightarrow 2^{x}$ définie, pour tout $A \subset X$, par $\Delta A=\{y \in Y \mid x \in A \Rightarrow\langle x, y\rangle<0\} . \Delta^{*} \Delta A$ coïncide alors avec l'intersection des demi-espaces ouverts homogènes contenant $A$. Ainsi, $\Delta^{*} \Delta A=X$ si $A$ n'est contenu dans aucun demi-espace ouvert homogène, soit, par exemple, si $0 \in A$. Ceci étant, la proposition suivante complète le lemme 2.5 et le corollaire $3.2 \mathrm{de}$ [34].

Proposition III.1.1. - Soit A une partie de $X$ incluse dans un demi-espace ouvert homogène. Le cône "evenly" convexe engendré par A, à savoir l'intersection de tous les cônes evenly convexes contenant $A$, coïncide avec $\Delta^{*} \Delta A$.

Prevve. - Soit $K$ le cône "evenly " convexe engendré par $A$. Puisque tout demiespace ouvert homogène est un cône "evenly " convexe, on a toujours $K \subset A^{*} \Delta A$. Dès lors, 0 ne peut appartenir à $K$ car on aurait alors $0 \in \Delta^{*} \Delta A$, soit encore $\Delta 0 \supset \Delta A$, d'où $\Delta A=\emptyset$ et $\Delta^{*} \Delta A=X$, ce qui est contraire à l'hypothèse selon laquelle $A$ est inclus dans un demi-espace ouvert homogène. Ainsi, $0 \notin K$ et, d'après [34], lemme $2.5, K$ est intersection de demi-espaces ouverts homogènes, d'où il résulte que $K \supset \Delta^{*} \Delta A$.

D'après (13), les fonctions $f$ dont les tranches sont fermées pour $\Delta^{*} \Delta$ sont celles pour lesquelles il existe $g \in \overline{\mathbb{R}}^{x}$ arec, pour tout $x \in X$,

$$
f(x)=-\inf \{g(y) \mid\langle x, y\rangle \geqslant 0\} .
$$

De là et de la proposition III.1.1, il résulte que ces fonctions coïncident avec les fonctions quasi "evenly" convexes homogènes propres.

Le théorème suivant est à comparer avec les récents résultats obtenus indépendamment par Martinez-Legaz [27].

THÉoRìm III.1.2. - Une fonction atteignant son maximum à l'origine est quasi "evenly " convexe homogène propre si et seulement si ses tranches sont des cônes "evenly" convexes.

Preuve. - La condition est évidemment nécessaire; elle est aussi suffisante car une fonction atteignant son maximum à l'origine et dont les tranches sont des cônes "evenly " convexes à des tranches fermées pour $\Delta^{*} \Delta$.

On a ici $\Delta^{*} 0=\Delta 0=\emptyset$, de sorte que, d'après prop. I.3.1 iv)

$$
0 \in \partial^{\Delta} f(a) \Leftrightarrow f(a)=\inf f
$$

La proposition suivante donne la structure du $\Delta$-quasidifférentiel. 
PRopositron IIT.1.3. - Une fonetion f est $\Delta$-quasidifférentiable en a si et seulement si il existe un demi-espace fermé homogène contenant a et sur lequel $f$ atteint son minimum en a. En outre, osf $f(a)$ est un cône "evenly" convexe.

Preuve. - Si $b \neq 0, b \in \partial^{\Delta} f(a)$ si et seulement si $f$ atteint en $a$ son minimum sur le demi-espace $\{x \mid\langle x, b\rangle \geqslant 0\}$.

Si $b=0$ et d'après (23), $f$ atteint en $a$ son minimum sur tout demi-espace fermé homogène contenant $a$. Enfin, d'après Th. I.3.2 ii), $\partial^{4} f(a)$ est l'intersection de $(\Delta a)^{c}$ qui est un demi-espace fermé homogène, done un cône "evenly " convexe, et de $\Delta T_{f(a)} f=\bigcap\{\Delta x \mid f(x)<f(a)\}$ qui est une intersection de demi-espaces ouverts bomogènes, ce qui permet de conclure.

ExeMrle. - Quasiconjugaison au sens de Passy et Prisman ([34], [35], [36]). A toute fonction quasiconvexe $g \in \overline{\mathbb{R}}^{x}$, PASSY et PRISMAn associent la fonction quasiconvexe homogène propre $f_{g} \in \overline{\mathbb{R}}^{X \times \mathbb{R}}$ définie par $f_{g}(x, \lambda)=g(x / \lambda)$ si $\lambda>0, f_{g}(x, \lambda)=$ $=\sup g$ si $\lambda \leqslant 0$. Pour étudier ce type de fonctions ils introduisent une conjugaison qui est un cas particulier de ce qui précède. Soit, en effet, la polarité $\Delta: 2^{X \times \mathbb{R}} \rightarrow 2^{X \times \mathbb{R}}$ définie, pour tout $A \subset X \times \mathbb{R}$, par $\Delta A=\{(y, s) \in Y \times \mathbb{R} \mid(x, r) \in A \Rightarrow\langle x, y\rangle+r s<0\}$. La conjuguée de $j \in \overline{\mathbb{R}}^{X \times \mathbb{R}}$ introduite par Passy et Prisman n'est autre que $f^{\Delta}(y, s)=$ $=-\inf \{f(x, r) \mid\langle x, y\rangle+r s \geqslant 0\}$. Le théorème 3.1 de [34] est alors un cas typique de la conjugaison par tranches.

\section{III.2. Quasiconjugaison au sens de Greenberg-Pierskalla ([22]).}

Soit la polarité $\Delta: 2^{X} \rightarrow 2^{X \times R}$ définie, pour tout $A \subset X$, par $\Delta A=\{(y, s) \in Y \times$ $\times \mathbb{R} \mid x \in A \Rightarrow\langle x, y\rangle\langle s\} . \Delta^{*}(y, s)$ est ici un demi-espace ouvert et $\Delta^{*} \Delta A$ est l'enveloppe "evenly " convexe de $A$. Les fonctions $f \in \overline{\mathbb{R}}^{x}$ telles que $f=f^{\Delta \Delta^{*}}$ sont donc les fonctions quasi "evenly " convexes. On a aussi $f^{A}(y, s)=-\inf \{f(x) \mid\langle x, y\rangle \geqslant s\}$ et, si $f_{s}^{G}$ désigne la s-polaire de $f$ au sens de [22], on a:

$$
f_{s}^{\theta}=s+f^{\Delta}(\cdot, s)
$$

Si $f^{\theta G}$ désigne la bipolaire quasiconvexe de [22], il vient $f^{\sigma G}=f^{\Delta \Delta^{*}}$. On obtient done une conjugaison exacte et symétrique pour les fonctions quasi "evenly" convexes (voir aussi [28]).

Notons qu'une base intersectionnelle pour l'opérateur $\Delta \Delta^{*}$ est ici constituée par les épigraphes stricts des formes linéaires $\langle x, \cdot\rangle$; ces épigraphes stricts sont des demi-espaces ouverts homogènes de $Y \times \mathbb{R}$, d'où il résulte que les parties fermées pour $\Delta \Delta^{*}$ sont des cônes "evenly" convexes.

THEORENE III.2.1. - Pour toute fonction g de $Y \times \overline{\mathbb{R}}$ dans $\mathbb{R}$, les propriétés i) et ii) sont équivatentes et impliquent iii).

i) $g=g^{d * A}$. 
ii) Il existe $f \in \overline{\mathbb{R}}^{x}$ telle que, pour tout $(y, s) \in Y \times \mathbb{R}$,

$$
g(y, s)=-\inf \{f(x) \mid\langle x, y\rangle \geqslant r\} \text {. }
$$

iii) g est quasi «evenly» convexe propre et, pour tout $y \in \bar{Y}, g(y, \cdot)$ est déeroissante.

Preuve. - Pour i) $\Leftrightarrow$ ii), voir (13). Pour ii) $\Rightarrow$ iii), le seul point délicat est de prouver que $g=f^{\Delta}$ est quasi "evenly" convexe. Or, pour tout $\lambda \in \mathbb{R}, \mathbb{T}_{\lambda} g=\mathbb{T}_{\lambda} f^{\Delta}$ est une partie fermée pour $\Delta \Delta^{*}$, donc un ensemble "evenly" convexe d'après la remarque qui précède ce théorème.

La proposition suivante montre que la projection sur $Y$ du $\Delta$-quasidifférentiel de $f$ en $a$, notée $P_{Y} \partial^{\Delta} f(a)$, coïncide avec le quasi-sous-différentiel au sens de [22] de $f$ en $a$, noté $\partial^{a} f(a)$.

Proposition III.2.2. - $\$ i f(a) \in \mathbb{R}$, il y a équivalence entre

i) $b \in \partial^{G} f(a)$;

ii) $(b,\langle a, b\rangle) \in \partial^{\Delta} f(a)$;

iii) $b \in P_{Y} \partial^{\Delta} f(a)$.

Preuve. - i) $\Leftrightarrow$ ii) car $b \in \partial^{a} f(a)$ signifie $f(a)+f_{\langle a, b\rangle}^{a}(b)=\langle a, b\rangle$, soit, d'après $(24), f^{\Delta}(b,\langle a, b\rangle)=-f(a)$ ce qui, compte tenu du fait que $(b,\langle a, b\rangle) \notin \Delta a$, permet de conclure. L'implication ii) $\Rightarrow$ iii) étant triviale, montrons que iii) $\Rightarrow$ ii). Soit done $(b, s) \in \partial^{4} f(a)$. On a $\langle a, b\rangle \geqslant s$ et, puisque $f^{\Delta}(b, \cdot)$ est décroissante, $f(a)=$ $=-f^{\Delta}(b, s) \leqslant-f^{\Delta}(b,\langle a, b\rangle) \leqslant f(a)$, d'où $f(a)+f_{\langle a, b\rangle}^{G}(b)=\langle a, b\rangle$.

\section{III.3. Regularisations quasiconvexes s.c.i.}

a) Fonctions dont les tranches sont des cônes convexes fermés. - Soit la polarité $\Delta: 2^{X} \rightarrow 2^{Y}$ définie, pour tout $A \subset X$, par $\Delta A=\{y \in Y \mid \sup \{\langle x, y\rangle \mid x \in A\} \leqslant 0\} . \Delta A$ est un cône convexe fermé et $\Delta^{*} \Delta A$ le cône convexe fermé engendré par $A$. (Les cônes dont il s'agit ont tous pour sommet l'origine). Le théorème suivant caractérise les fonctions qui coïncident avec leur biconjuguée.

THÉoRìine III.3.1. - Pour toute fonotion $f \in \overline{\mathbb{R}}^{x}$, il y a équivalence entre

i) Les tranches de $f$ sont des cônes convexes fermés.

ii) Il existe $g \in \overline{\mathbb{R}}^{y}$ telle que, pour tout $x \in X$,

$$
f(x)=-\inf \{g(y) \mid\langle x, y\rangle>0\} .
$$

iii) $f$ est quasiconvexe homogène s.c.i. et $f(0)=-\infty$. 
Preuve. - Pour i) $\Leftrightarrow$ ii), voir (13). Pour ii) $\Rightarrow$ iii) on vérifie sans peine que $f$ est homogène et que $f(0)=-\infty$; en, outre, pour tout $\lambda \in \mathbb{R}, \mathbb{T}_{\lambda} f={ }^{\top} T_{\lambda} g^{\Delta^{*}}$ est une partie fermée pour $\Delta \Delta^{*}$, à savoir un cône convexe fermé. Pour iii) $\Rightarrow$;) montrons que, pour tout $\lambda \in \mathbb{R}, \mathbb{T}_{\lambda} f$ est un cône convexe fermé. $f$ étant quasiconvexe homogène, $\mathbb{T}_{\lambda} f$ est un cône convexe non vide du fait que $f(0)=-\infty$ et fermé du fait que $f$ est s.e.i.

b) Eutude d'une conjugaison exacte et symétrique pour les fonetions quasiconvexes s.o.i. - Soit la polarité $\Delta: 2^{X} \rightarrow 2^{Y \times \mathbb{R}}$ définie, pour tout $A \subset X$, par $\Delta A=\{(y, s) \in$ $\in I \times \mathbb{R} \mid \sup \{\langle x, y\rangle \mid x \in A\} \leqslant s\}$. $\Delta^{*}(y, s)$ est ici un demi-espace fermé d'où il résulte que $\Delta^{*} \Delta A$ est l'enveloppe convexe fermée, $\overline{c o} A$, de $A$. Les fonctions $f \in \overline{\mathbb{R}}^{x}$ telles que $f=f^{\Delta \Delta^{*}}$ sont done les fonctions quasiconvexes s.c.i. Avant de passer à l'étude du $\Delta$-quasidifférentiel, remarquons que si $\alpha_{A}$ désigne la fonction d'appui de $A$, à savoir $\alpha_{A}(y)=\sup \{\langle x, y\rangle \mid x \in A\}$, on a:

$$
\Delta A=\operatorname{Epi} \alpha_{A}
$$

Proposition III.3.2. - $\$ i\langle a, b\rangle>r$ on a l'équivalence:

$$
(b, r) \in \partial^{d} f(a) \Leftrightarrow \forall s, \quad\langle a, b\rangle>s \geqslant r:(b, s) \in \partial^{A} f(a) .
$$

Preuve. - Si $r \leqslant s\left\langle\langle a, b\rangle\right.$ et si $(b, r) \in \partial^{\Delta} f(a)$, il vient $f(a)=\inf \{f(x) \mid\langle x, b\rangle>r\} \leqslant$ $\leqslant \inf \{f(x) \mid\langle x, b\rangle>s\} \leqslant f(a)$, d'où $(b, s) \in \partial^{A} f(a)$.

PRoposIrTon III.3.3. - Les propriétés suivantes sont équivalentes:

i) f atteint en a un minimum global;

ii) $Y=P_{Y} \hat{\partial} \Delta f(a)$;

iii) $0 \in P_{y} \partial^{4} f(a)$.

PReUve. - Pour i $) \Rightarrow$ ii $)$, soit $y \in Y$ et $r\langle\langle a, y\rangle$; il vient inf $f \leqslant \inf \{f(x) \mid\langle x, y\rangle>r\}$ $\leqslant f(a)=\inf f$. L'implication ii) $\Rightarrow$ iii) est évidente. Pour iii) $\Rightarrow$ i) il existe $r<0$ tel que $f(a)=\inf \{f(x) \mid\langle x, 0\rangle>r\}=\inf f$.

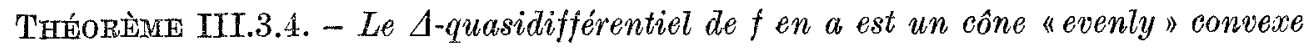
épointé dont la projection sur $\bar{Y}$ est incluse dans le tangentiel de Crouzeic de $f$ en a, noté $T f(a)([7])$.

Preuve. - $(\Delta a)^{c}$ est l'hypographe strict de la forme linéaire $\langle a, \cdot\rangle$, done un demiespace homogène ouvert de $Y \times \mathbb{R}$. Par ailleurs, d'après (25), $\Delta T_{f(a)} f$ est l'épigraphe de la fonction d'appui de $T_{f(a)} f$, e'est-à-dire un cône convexe fermé de $Y \times \mathbb{R}$. De 
Th. I.4.2. ii) résulte alors que $\partial^{4} f(a)$ est un cône "evenly" convexe épointé. En outre, si $(b, r) \in \partial^{\triangleleft} f(a)$, on obtient (Th. I.4.2.i)) $\langle a, b\rangle>r$ et, pour tout $\lambda<f(a)$, $\mathbb{T}_{i} f \subset \Delta^{*}(b, r)$. Ceei implique que, pour tout $\lambda<f(a), \sup \{\langle x-a, b\rangle \mid f(x) \leqslant \lambda\}<0$, soit exactement $b \in T f(a)$.

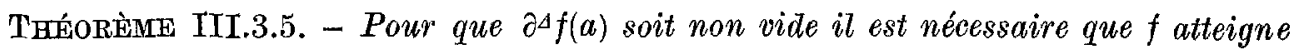
en a un minimum local. Si cette condition est remplie et si $X=Y=\mathbb{R}$ avec $\langle x, y\rangle=$ $=x y$ on a l'égalité $P_{Y} \partial^{d} f(a)=\top f(a)$.

Preuve. - Si a n'est pas minimum local, il existe une suite généralisée $\left(x_{i}\right)$ qui converge vers $a$ et telle que, pour tout $i, f\left(x_{i}\right)<f(a)$. En supposant que $(b, r) \in \partial^{d} f(a)$ on aurait alors, pour tout $i,\langle a, b\rangle>r$ et $\left\langle x_{i}, b\right\rangle \leqslant r$ ce qui est absurde. Soit maintenant $X=Y=\mathbb{R}$ et $\langle x, y\rangle=x y$. Prenons $b \in T f) a$ ) et $\lambda\langle f(a)$. Puisque $a$ est minimum local de $f$ il existe $\alpha>0$ tel que $f(x)<\lambda$ implique $|x-a|>\alpha$. Soit alors $f(x)<\lambda$ et supposons tout d'abord $b>0$. Si $x-a>\alpha$ alors $x b-a b>\alpha b$ mais, par hypothèse, $f(x)<\lambda$ implique $x b-a b<0$; on ne peut donc pas avoir $x-a>\alpha$. Si $a-x>\alpha$ alors $x b-a b<-\alpha b$ et $(b, a b-\alpha b) \in \partial^{4} f(a)$. Prenons maintenant $b<0$. $x-a>\alpha$ implique alors $x b-a b<\alpha b$ et $(b, a b+\alpha b) \in \partial d f(a)$. Si $a-x>\alpha$ alors $x b-a b>-\alpha b$; d'autre part, $f(x)<\lambda$ implique $x b-a b<0$, ce qui est absurde. Supposons enfin $b=0$; il s'ensuit que $f$ atteint en $a$ un minimum global et qu'alors (Prop. III.3.3) $\left\lceil f(a)=\mathbb{R}=P_{\mathbb{R}} \partial^{d} f(a)\right.$.

Pour caractériser les fonctions $g$ de $Y \times \overline{\mathbb{R}}$ dans $\overline{\mathbb{R}}$ telles que $g=g^{\Delta^{*} \Delta}$, notons tout d'abord qu'une base intersectionnelle pour l'opérateur $\Delta \Delta^{*}$ est constituée par les Epi $\langle x, \cdot\rangle$ avec $x \in X$. Il en résulte que les parties fermées pour $\Delta \Delta^{*}$ ne sont autres que les épigraphes des fonctions sous-linéaires s.c.i. Plus précisément, si $B \subset Y \times \mathbb{R}$ et si on désigne par Inf $B$ la fonction de $Y$ dans $\mathbb{R}$ définie par $(\operatorname{Inf} B)(y)=$ $=\inf \{s \mid(y, s) \in B\}$ on peut énoncer:

Propostrion III.3.6. - Pour tout $B \subset Y \times \mathbb{R}, \Delta \Delta^{*} B$ est l'épigraphe de la régularisée sous-linéaire s.c.i. de $\operatorname{Inf} B$.

Preuvk. $-(w, s) \in \Delta \Delta^{*} B$ équivaut à $\Delta^{*}(w, s) \supset \Delta^{*} B$, soit encore, successivement, à :

$$
\begin{gathered}
((y, r) \in B \Rightarrow\langle x, y\rangle \leqslant r) \Rightarrow(\langle x, w\rangle \leqslant s) ; \\
(\langle x, \cdot\rangle \leqslant \operatorname{Inf} B) \Rightarrow(\langle x, w\rangle \leqslant s) ; \quad \sup \{\langle x, w\rangle\langle x, \cdot\rangle \leqslant \operatorname{Inf} B\} \leqslant s .
\end{gathered}
$$

Le membre de gauche de la dernière inégalité n'est autre que la valeur en $w$ de la régularisée sous-linéaire s.c.i. de $\operatorname{Inf} B$.

THÉORÈME III.3.7. - Pour toute fonction $g: Y \times \mathbb{R} \rightarrow \overline{\mathbb{R}}$, il y a équivalence entre

i) Les tranches de $g$ sont des épigraphes de fonctions sous-linéaires s.c.i. 
ii) Il existe $f \in \overline{\mathbb{R}}^{x}$ telle que, pour tout $(y, s) \in Y \times \mathbb{R}$,

$$
g(y, s)=-\inf \{f(x) \mid\langle x, y\rangle>s\} .
$$

iii) $g$ est quasiconvexe homogène s.c.i., $g(0,0)=-\infty$, pour tout $y \in Y g(y, \cdot)$ est décroissanie.

Preuve. - i) $\Leftrightarrow$ ii) résulte de la théorie générale et on vérifie aisément que ii) $\Rightarrow$ iii). Si $g$ vérifie iii) il est facile de voir que, pour tout $\lambda \in \mathbb{R}$, $\mathbb{T}_{\lambda} g$ est un cône convexe fermé; pour obtenir i) il reste à prouver que $\mathbb{T}_{\lambda} g$ est l'épigraphe d'une fonction $\varphi \in \overline{\mathbb{R}}^{Y}$ qui sera nécessairement sous-linéaire s.c.i.; pour cela posons $\varphi(y)=$ $=\inf \{s \mid g(y, s) \leqslant \lambda\}$ et montrons que $g(y, r) \leqslant \lambda$ équivaut à $\varphi(y) \leqslant r$. La fonetion $g(y, \cdot)$ étant décroissante s.c.i., cette équivalence résulte d'un fait général donné par le lemme suivant.

LEMME III.3.8. - Soit f et g deux fonctions de $\mathbb{R}$ dans $\overline{\mathbb{R}}$ telles que, pour tout $\lambda \in \mathbb{R}$, $g(\lambda)=\inf \{s \mid f(s) \leqslant \lambda\}$. On a alors, pour tous réels $r, \lambda, f(r) \leqslant \lambda \Leftrightarrow g(\lambda) \leqslant r$. En partioulier, $g$ est décroissante s.c.i.

Preuve. - Par définition de $g, f(r) \leqslant \lambda$ implique $g(\lambda) \leqslant r$. Réciproquement, supposons $f(r)>\lambda$. Puisque $f$ est s.c.i., il existe $\varepsilon>0$ tel que $|s-r|<\varepsilon$ implique $f(s)>\lambda$. Or, $f$ étant décroissante, on ne peut avoir simultanément $f(s) \leqslant \lambda$ et $r-s \geqslant \varepsilon$. On voit done que $f(s) \leqslant \lambda$ implique $s-r \geqslant \varepsilon$, d'où il résulte que $g(\lambda) \geqslant r+\varepsilon . g$ est alors s.c.i. car, pour tout $r \in \mathbb{R}, \mathbb{T}_{r} g=[f(r),+\infty[$.

c) Conjugaison au sens de Flachs et Pollatscheh ([19], [20], [21]). - Etant donné $\varphi: X \times \mathbb{R} \rightarrow \overline{\mathbb{R}}$ et $\psi: Y \times \mathbb{R} \rightarrow \overline{\mathbb{R}}$, FLACHS et PoLdatsdneK considèrent les fonetions $\varphi^{0}: Y \times \mathbb{R} \rightarrow \overline{\mathbb{R}}$ et $\psi^{*}: X \times \mathbb{R} \rightarrow \overline{\mathbb{R}}$ définies par $\varphi^{0}(y, s)=\inf \{\varphi(x, t)|\langle x, y\rangle-t\rangle s\}$, $\psi^{*}(x, t)=\sup \{\psi(y, s)|\langle x, y\rangle-s\rangle t\}$. Soit alors la polarité $\Delta: 2^{X \times \mathbb{R}} \rightarrow 2^{\mathbf{Y} \times \mathbb{R}}$ définie, pour tout $A \subset X \times \mathbb{R}$, par $\Delta A=\{(y, s) \in Y \times \mathbb{R} \mid(x, t) \in A \Rightarrow\langle x, y\rangle-t \leqslant s\}$. On obtient ainsi $\varphi^{0}=-\varphi^{\Delta} ; \psi^{*}=(-\psi)^{\Delta^{*}} ; \varphi^{0 *}=\varphi^{\Delta \Delta^{*}}$. Dans [19], théorème 2, des conditions suffisantes sont données pour que $\varphi^{0 *}$ et $\varphi$ coïncident. Grâce à la polarité $\Delta$ nous allons caractériser les fonctions $\varphi$ telles que $\varphi=\varphi^{0 *}$.

Proposimion III.3.9. - Pour tout $A \subset X \times \mathbb{R}, \Delta^{*} \Delta A$ coincide avec l'épigraphe de la régularisée convexe s.c.i. de la fonction $x \mapsto(\operatorname{Inf} A)(x)=\inf \{t \mid(x, t) \in A\}$.

Prevve. $-(\bar{x}, \bar{t}) \in \Delta^{*} \Delta A$ équivaut à $\Delta(\bar{x}, \bar{t}) \supset \Delta A$, soit encore, successivament à:

$$
\begin{aligned}
& ((x, t) \in A \Rightarrow\langle x, y\rangle-s \leqslant t) \Rightarrow(\langle\vec{x}, y\rangle-s \leqslant \bar{t}) ; \\
& (\langle\cdot, y\rangle-s \leqslant \operatorname{Inf} A) \Rightarrow(\langle\bar{x}, y\rangle-s \leqslant \bar{t}) ;
\end{aligned}
$$

$\sup \{\langle\bar{x}, y\rangle-s \mid\langle\cdot, y\rangle-s \leqslant \operatorname{Inf} A\} \leqslant \bar{t} ;$ or, le membre de gauche de cette dernière inégalité n'est autre que la valeur en $\bar{x}$ de la régularisée convexe s.c.i. de Inf $A$. 
Le théorème suivant caractérise les fonctions dont les tranches sont des épigraphes de fonctions convexes s.c.i.

Thtók̀̀me III.3.10. - Pour toute fonction $\varphi: X \times \mathbb{R} \rightarrow \overrightarrow{\mathbb{R}}$ il $y$ a équivalente entre

i) Il existe $\psi: Y \times \mathbb{R} \rightarrow \overline{\mathbb{R}}$ telle que, pour tout $(x, t) \in X \times \mathbb{R}$,

$$
\varphi(x, t)=-\inf \{\psi(y, s) \mid\langle x, y\rangle-s>t\} .
$$

ii) $\varphi$ est quasiconvexe s.c.i. et, pour tout $x \in X, \varphi(x, \cdot)$ décroissante.

Precve. - i) $\Rightarrow$ ii). D'après la proposition III.3.9, les tranches de $\varphi$ sont des convexes fermés; par ailleurs, $\varphi(x, \cdot)$ est visiblement décroissante.

ii) $\Rightarrow$ i). Soit $\lambda \in \mathbb{R} ; \varphi$ étant quasiconvexe s.c.i., $\mathbb{T}_{\lambda} \varphi$ est convexe fermé; reste à prouver que ' $\mathrm{T}_{\lambda} \varphi$ est l'épigraphe d'une fonction qui sera nécessairement convexe s.c.i. Pour cela, posons $k(x)=\inf \{t \in \mathbb{R} \mid \varphi(x, t) \leqslant \lambda\}$. La fonction $\varphi(x, \cdot)$ étant décroissante s.c.i., le lemme III.3.8 nous donne l'équivalence $\varphi(x, r) \leqslant \lambda \Leftrightarrow k(x) \leqslant r$, soit précisément $\mathbb{T}_{\lambda \varphi}=$ Epi $k$.

III.4. Autres conjugaisons utiles en quasiconvexite.

a) Conjugaisons d'Atteia-El Qortobi ([2], [13]). - Etant donné $f \in \overline{\mathbb{R}}^{x}$, Atteia et EL QoRToBI considèrent fo et $f^{\nabla}$ dans $\overline{\mathbb{R}}^{P}$ définies par $f^{0}(y)=-\inf \{f(x) \mid\langle x, y\rangle>1\}$ et $f^{\nabla}(y)=-\inf \{f(x) \mid\langle x, y\rangle<1\}$. Ils montrent que $\max \left(f^{00}, f^{\nabla \nabla}\right)$ est la régularisée quasiconvexe s.c.i. de $f$. Or, chacune des fonctions $f^{0}$ et $f^{\nabla}$ s'interprête comme conjuguée de $f$ par rapport à une polarité. Ainsi, soit $\Delta: 2^{x} \rightarrow 2^{Y}$ définie par $\Delta A=$ $=\{y \in Y \mid x \in A \Rightarrow\langle x, y\rangle \leqslant 1\}$. On a alors $f^{0}=f^{\Delta}, f^{00}=f^{\Delta \Delta^{*}}$ et, d'après le théorème des bipolaires, $\Delta^{*} \Delta A=\overline{\mathrm{co}}(A \cup\{0\})$. Done, les tranches de $f$ sont fermées pour $\Delta^{*} \Delta$ si et seulement si $f$ est quasiconvexe s.c.i. et prend la valeur $-\infty$ en 0 ; d'où:

THÉoRغ̀ME III.4.1. - Les propriétés suivantes sont équivalentes:

i) $f=f^{00}$.

ii) Il existe $g \in \overline{\mathbb{R}}^{T}$ telle que, pour tout $x \in X, f(x)=-\inf _{\langle x, y\rangle>1} g(y)$.

iii) $f$ est quasiconvexe s.c.i. et $f(0)=-\infty$.

Soit maintenant la polarité $\Omega: 2^{x} \rightarrow 2^{X}$ donnée par $\Omega(A)=\{y \in Y \mid x \in A \Rightarrow\langle x, y\rangle \geqslant 1\}$. On a $f^{\nabla}=f^{\Omega}, f^{\nabla \nabla}=f^{\Omega \Omega^{*}}$ et, d'après [13] Th. 1.1,

$$
\Omega^{*} \Omega A=\overline{\bigcup_{\beta \geqslant 1} \beta A} \quad \text { pour tout convexe fermé } A \text { ne contenant pas } 0 .
$$


THÉORł̀mE III.4.2. - Les propriétés suivantes sont équivalentes:

i) $f=f^{\nabla \nabla}$.

ii) Il existe $g \in \overline{\mathbb{R}}^{x}$ telle que, pour tout $x \in X$,

$$
f(x)=-\inf \{g(y) \mid\langle x, y\rangle<1\} .
$$

iii) $f$ est quasiconvexe s.c.i. et $f(\alpha x) \geqslant f(x)$ pour tous $x \in X$ et $\alpha \in[0,1]$.

PreUve. - ii) $\Rightarrow$ iii) car, pour tout $\lambda \in \mathbb{R}, \mathbb{T}_{\lambda} f=\{x \mid\langle x, y\rangle\langle 1 \Rightarrow g(y) \geqslant-\hat{\lambda}\}=$ $=\bigcap_{g(y)<-\lambda}\{x \mid\langle x, y\rangle \geqslant 1\}$ est convexe fermé. En outre, si $x \in X$ et $\alpha \in[0,1],\langle x, y\rangle<1$ implique $\langle\alpha x, y\rangle<1$ de sorte que $f(x) \leqslant f(\alpha x)$. Pour iii) $\Rightarrow$ i) notons qu'en prenant $\alpha=0$ il vient $f(0)=\sup f$. Soit alors $\lambda \in \mathbb{R} ;$ si $\lambda \geqslant f(0)$ alors $\mathbb{T}_{\lambda} f=X$; si $\lambda<f(0)$, $\mathbf{T}_{\lambda} f$ est un conveze fermé qui ne contient pas 0 . D'après (26), il nous suffit done de montrer que $\mathbb{T}_{\lambda} f=\bigcup_{\beta \geqslant 1} \beta \mathbb{T}_{\lambda} f$. L'inclusion $c$ étant triviale, soit $\beta \geqslant 1$ et $x \in \beta \mathbb{T}_{\lambda} f$; $x$ s'écrit $\beta x^{\prime}$ avec $f\left(x^{\prime}\right) \leqslant \lambda$. Mais $f(x)=f\left(\beta x^{\prime}\right) \leqslant f\left(1 / \beta\left(\beta x^{\prime}\right)\right) \leqslant \lambda$, ce qui permet de conclure.

b) Pseudo-conjugaison de I. Singer ([40]). - Singer introduit une notion de pseudo-dualité permettant de définir le pseudo-dual d'un problème donné avec un saut de dualité moindre que dans la théorie de Gremerberg et Pierskalla ([22]). En fait, cette pseudo-dualité découle de la polarité $\Delta: 2^{X} \rightarrow 2^{Y \times \mathbb{R}}$ définie par $\Delta A=$ $=\{(y, r) \in Y \times \mathbb{R} \mid x \in A \Rightarrow\langle x, y\rangle \neq r\}$. Une base intersectionnelle pour $\Delta^{*} \Delta$ est constituée par les complémentaires d'hyperplans de $X$, de sorte que $f=f^{\Delta \Delta^{*}}$ si et seulement si les tranches de $f$ sont intersections de complémentaires d'hyperplans. On a aussi

$$
f^{4}(y, r)=-\inf \{f(x) \mid\langle x, y\rangle=r\}=f_{r}^{\Pi}(y)-r
$$

où $f_{r}^{H}$ désigne la r-pseudo-conjuguée de $f$ au sens de [40].

En outre, $f^{\Delta 4^{*}}=f^{H H}$ où $f^{H H}$ désigne la pseudo-biconjuguée de $f([40])$. En notant $\partial^{H} f(a)$ le pseudo-sous-différentiel de $f$ en a ([40]), on obtient:

Proposition III.4.3. - $\$ i f(a) \in \mathbb{R}$, il y a équivalence entre
i) $b \in \partial^{y} f(a)$;
ii) $(b,\langle a, b\rangle) \in \partial^{A} f(a)$;
iii) $b \in P_{Y} \partial^{\Delta} f(a)$.

PREUve. - i) $\Leftrightarrow$ ii) car $b \in \partial^{H} f(a)$ signifie $f(a)+f_{\langle a, b\rangle}^{\text {H }}(b)=\langle a, b\rangle$ soit, d'après $(27), f^{\Delta}(b,\langle a, b\rangle)=-f(a)$ et qu'en outre $(b,\langle a, b\rangle) \notin \Delta a$. L'implication ii) $\Rightarrow$ iii) est triviale; pour iii) $\Rightarrow \mathrm{i})$, soit $(b, r) \in \partial^{\Delta} f(a)$. On a aussitôt $r=\langle a, b\rangle$ et, d'après $(27), f(a)=-f_{\langle a, b\rangle}^{H}(b)-\langle a, b\rangle$, ce qui permet de conclure. 
c) Etude d'une conjugaison quasiconvexe exacte et symétrique. - Nous avons déjà introduit en II.1. b) une telle conjugaison. En voici une autre basée sur [26]. Dans [26], Martinez Legaz définit une conjugaison exacte pour les fonetions quasiconvexes de $\mathbb{R}^{n}$ dans $\overline{\mathbb{R}}$ en se servant de l'ordre lexicographique $\leqslant_{L}$ sur $\overline{\mathbb{R}}^{n}$. Le résultat clé est le théorème de séparation suivant: Si $C$ est une partie convexe de $\mathbb{R}^{n}$ et si $x_{0} \notin C$, il existe une matrice carrée d'ordre $n$ à coefficients réels $M(M \in \Omega)$ et $t \in \overline{\mathbb{R}}^{n}$ tels que $M x_{0} \geqslant_{L} t$ et, pour tout $x \in C, M x<_{L} t$. Ce théorème fournit une base intersectionnelle pour les parties convexes de $\mathbb{R}^{n}$ et nous conduit à considérer la polarité $\Delta$ de $2^{\mathbb{R}^{n}}$ dans $2^{\Omega \times \overline{\mathbb{R}}^{n}}$ définie, pour tout $A \subset \mathbb{R}^{n}$, par $\Delta A=\{(M, t) \in \Omega \times$ $\left.\times \overline{\mathbb{R}}^{n} \mid x \in A \Rightarrow M x<_{L} t\right\}$. On a alors $\Delta^{*}(M, t)=\left\{x \in \mathbb{R}^{n} \mid M x<_{L} t\right\}$ et, d'après le théorème ci-dessus, $\Delta^{*} \Delta A=\operatorname{co} A$. Si $f \in \overline{\mathbb{R}}^{\mathbb{R}^{n}}, f^{\Delta \Delta^{*}}$ est donc, par construction, la régularisée quasiconvexe de $f$. Pour arriver au même résultat, Martinkz LEgazintroduit l'ensemble $H$ des fonctions de $\mathbb{R}^{n}$ dans $\overline{\mathrm{R}}$ croissantes pour $\leqslant_{L}$ et il définit successivement $f^{c} \in H^{\Omega}$ par $f^{c}(M)=\sup \{h \in H \mid h \circ M \leqslant f\}$ et $f^{c \nabla} \in \overline{\mathbb{R}}^{\mathbb{R}^{n}}$ par $f^{\circ \nabla}=\sup _{M} f^{c}(M) \circ M$. $f^{c \nabla}$ est encore la régularisée quasiconvexe de $f$, mais le procédé n'est plus du tout symétrique.

Proposition III.4.4. - La régularisée quasiconvese $f^{\Delta \Delta^{*}}$ de $f \in \overline{\mathbb{R}}^{\mathbb{R}^{n}}$ s'écrit:

$$
f^{\Delta \Delta^{*}}(a)=\sup _{M \in \Omega} \inf \left\{f(x) \mid M(x-a) \geqslant{ }_{L} 0\right\}
$$

PREuve. - L'ordre $\leqslant_{L}$ étant total sur $\overline{\mathbb{R}}^{n}, f^{\Delta \Delta^{*}}(a)$ s'écrit, d'après (18),

$$
\sup _{M} \sup _{t \leqslant L M a} \inf \left\{f(x) \mid M x \geqslant_{L} t\right\}
$$

La fonction $t \mapsto \inf \left\{f(x) \mid M x \geqslant_{L} t\right\}$ étant croissante pour $\leqslant_{L}$ sur $\mathbb{R}^{n}$, il vient $f^{\Delta \Delta^{*}}(a)=$ $=\sup _{M} \inf \left\{f(x) \mid M x \geqslant_{L} M a\right\}$. La compatibilité de $\leqslant_{L}$ avec l'addition permet alors de conclure.

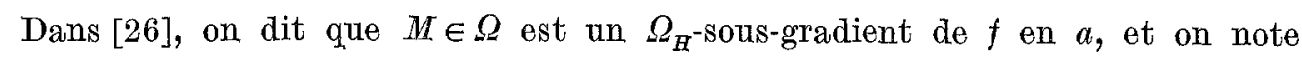
$M \in \partial_{H}^{\Omega} f(a)$, s'il existe $h \in H$ telle que $h \circ M \leqslant f$ et $(h \circ M)(a)=f(a)$. Il est prouvé que $M \in \partial_{H}^{\Omega} f(a)$ si et seulement si inf $\left\{f(x) \mid M x \geqslant_{L} M a\right\}=f(a)$ et que si $f$ est quasiconvexe, $\partial_{H}^{\Omega} f(a)$ n'est jamais vide.

PropostTIoN III.4.5. - $\partial_{H}^{\Omega} f(a)$ coïncide avec la projection sur $\Omega$ de $\partial^{\Delta} f(a)$.

Preuve. - Si $(M, t) \in \partial^{\Delta} f(a)$, il vient $M a \geqslant_{L} t$ et

$$
f(a)=f^{\Delta \Delta^{*}}(a)=\inf \left\{f(x) \mid M x \geqslant_{L} t\right\} .
$$

On a alors,

$$
f(a) \leqslant \inf \left\{f(x) \mid M x \geqslant_{L} M a\right\} \leqslant f^{\Delta \Delta^{*}}(a)
$$


(la dernière inégalité résultant de prop. III.4.4); on a donc bien $M \in \partial_{H}^{g} f(a)$. Réciproquement, si $f(a)=\inf \left\{f(x) \mid M x \geqslant_{L} M a\right\}$ on a tout de suite $(M, M a) \in \hat{o}^{\Delta} f(a)$.

d) Dualité en économie ([8] et références incluses). - On désigne par $K$ l'orthant positif de $\mathbb{R}^{n}$ et par $\langle$,$\rangle le produit scalaire de \mathbb{R}^{n}$. Etant donné une fonction $u$ de $K$ dans $\overline{\mathbb{R}}$ on utilise en économie les fonctions $v$ et $w$ définies sur $K$ par

$$
v(y)=\inf \{u(x) \mid x \in \mathbb{K} \text { et }\langle x, y\rangle \leqslant 1\} ; \quad w(x)=\sup \{v(y) \mid y \in \mathbb{K} \text { et }\langle x, y\rangle \leqslant 1\} .
$$

Il est alors utile de comparex $w$ à $u$.

Pour interpréter $v$ et $w$ considérons la polarité

$$
\Delta: 2^{K} \rightarrow 2^{\mathbb{R}}, \quad \Delta A=\{y \in \mathbb{R} \mid x \in A \Rightarrow\langle x, y\rangle>1\} .
$$

On a alors $v=-u^{\Delta}$ et $w=u^{\Delta \Delta^{*}}$.

Il est done immédiat que $w \leqslant u$ et que $w=u$ si et seulement si les tranches de $u$ sont intersections d'ensembles du type $\{x \in \mathbb{K} \mid\langle x, y\rangle>1\}$ avec $y \in \mathbb{K}$. D'après Th. I.3.3. iii), une condition nécessaire et suffisante pourque $u(a)=w(a)$ est

$$
\forall \lambda<u(a), \quad \exists y \in K \mid\langle a, y\rangle \leqslant 1 \quad \text { et } \quad v(y) \geqslant \lambda .
$$

En outre, le problème de la quasi 4 -différentiabilité de $u$ équivaut à la recherche de $y$ de $K$ tels que le problème

$$
\inf \{u(x) \mid x \in K \text { et }\langle x, y\rangle \leqslant 1\}
$$

ait au moins une solution dans $K$. Si $a$ est une telle solution, la prop. I.3.1. iii) nous dit que $a \in \partial^{\Delta^{*}} u_{i}^{A}(y)$ ce qui signifie que $y$ est solution du problème

$$
\sup \{v(x) \mid x \in K \text { et }\langle a, x\rangle \leqslant 1\} \text {. }
$$

\section{IV. - Applications à l'optimisation.}

\section{IV.1. Polarité associée d une function marginale.}

Soit $X, Z$ deux ensembles, $\Gamma: Z Z \rightarrow X$ une multiapplication, $f \in \overline{\mathrm{R}}^{X}$ et considé rons la fonction marginale $m$ définie sur $Z$ par $m(z)=\inf _{x \in \Gamma z} f(x)$. Il arrive qu'on ait besoin de montrer que les tranches de $m$ sont fermées pour un opérateur donné ( $m$ s.c.i., quasiconveze, etc.). Pour faciliter l'étude de $m$ introduisons la polarité $\Delta: 2^{Z} \rightarrow 2^{\mathbb{R}}$ définie, pour tout $A \subset Z$, par $\Delta A=\{\lambda \in \mathbb{R}|\forall z \in A, \exists x \in \Gamma z| f(x) \leqslant \lambda\}$. Une base intersectionnelle $d \mathrm{e} \Delta^{*} \Delta$ est ici constituée par les $\Delta * \lambda=\left\{z \mid \Gamma_{z} \cap \mathbb{T}_{R} f \neq \emptyset\right\}$ avec $\lambda \in \mathbb{R}$. En fait, $\Delta^{*} \lambda$ n'est autre que le domaine de la multiapplication composée Epifor déjà utilisée par Dolecki pour l'étude de $m$ ([10]). 
Proposition IV.1.1. - On a toujours $m=m^{\Delta \Delta^{*}}$.

Preuve. - Pour tout $\lambda \in \mathbb{R}$ il vient: $\mathbf{T}_{\lambda} m=\{z|\forall \varepsilon>0, \exists s x| x \in \Gamma z$ et $f(x) \leqslant \lambda+\varepsilon\}=$ $=\bigcap_{\varepsilon>0}\left\{z \mid \Gamma z \cap \mathbb{T}_{\lambda+\varepsilon} f \neq \emptyset\left\{=\bigcap_{\varepsilon>0} \Delta^{*}(\lambda+\varepsilon)\right.\right.$.

CoRollatre IV.1.2. - Pour que les tranches de m soient fermées pour un opérateur de fermeture donné, il est suffisant que, pour tout $\lambda \in \mathbf{R}, \Delta^{*} \lambda$ le soit.

Prevve. - D'après prop. IV.1.1, les tranches de $m$ sont intersections de $\Delta^{*} \lambda$.

ExeMpless. $-\Gamma$ s.c.s. et $f$ s.c.i. impliquent $\Delta^{*} \lambda$ fermé et $m$ s.c.i. ([9]). $\Gamma$ convexe et $f$ quasiconvexe impliquent $\Delta^{*} \lambda$ convexe et $m$ quasiconvexe.

La fonction $m$ est souvent donnée sous la forme $m(z)=\inf _{x \in X} \varphi(z, x)$ où $\phi$ est une fonction de $Z \times X$ dans $\overline{\mathbb{R}}$; soit alors la polarité $\Omega: 2^{Z} \rightarrow 2^{\mathbb{R}}$ définie par $\Omega A=$ $=\{\lambda \in \mathbb{R}|\forall z \in A, \exists x \in X| \varphi(z, \infty) \leqslant \lambda\}$. Ici encore $m=m^{\Omega \Omega^{*}}$.

Proposition IV.1.3. - On suppose que $Z$ est un espace vectoriel ordonné réticulé et que, pour tout $(x, z) \in X \times Z, \varphi(\cdot, x)$ est quasiconvexe et $\varphi(z, \cdot)$ eroissante (resp. décroissante). Dans ces conditions, pour tout $\lambda \in \mathbb{R}, \Omega^{*} \lambda$ est convexe et $m$ quasiconvexe.

Preuve. - Soit $\theta \in[0,1]$ et $z, z^{\prime} \in \Omega^{*} \lambda$; il existe $x, x^{r}$ dans $X$ tels que $\varphi(z, x) \leqslant \lambda$ et $\varphi\left(z^{\prime}, x^{\prime}\right) \leqslant \lambda$. Posant $y=\inf \left(x, x^{\prime}\right)$, il vient:

$$
\begin{aligned}
\varphi\left(\theta z+(1-\theta) z^{\prime}, y\right) & \leqslant \max \left\{\varphi(z, y), \varphi\left(z^{\prime}, y\right)\right\} \quad \text { car } \varphi(\cdot, y) \text { est quasiconvexe } \\
& \leqslant \max \left\{\varphi(z, x), \varphi\left(z^{\prime}, x^{\prime}\right)\right\} \\
& \leqslant \lambda
\end{aligned}
$$

Ainsi, $\theta z+(1-\theta) z^{\prime} \in \Omega^{*} \lambda$.

EXEMPLE. - Soit le problème de minimisation $\inf _{g(x) \leqslant 0} f(x)$ où $f \in \overline{\mathbb{R}}^{x}$ et $g \in Z^{x}$. Posons $\varphi(z, x)=f(x)$ si $g(x) \leqslant z,+\infty$ sinon.

Alors $m(z)=\inf _{g(x) \leqslant z} f(x)=\inf _{i:} \varphi(z, x)$. En appliquant la proposition précédente, on peut montrer que pour que $m$ soit quasiconvexe il suffit que $f$ et $g$ soient croissantes ou quasiconvexes.

REMARQUE. - On sait ([7]) que si $\varphi$ est quasiconvexe, $m$ l'est aussi mais on notera que $m$ peut très bien être quasiconvexe sans que $\varphi$ le soit ([41]).

IV.2. Programme dual relatif à une polarité.

Nous allons ici exploiter l'approche perturbationnelle de la dualité ([3], [25], $[37],[39], \ldots)$. Cette approche consiste à plonger le problème initial dans une famille 
paramétrée de problèmes et de définir ainsi une fonction marginale dont la valeur en un point précis redonne le problème initial. Nous définissons alors le problème dual par l'expression de la biconjuguée par tranches de la fonction marginale au point considéré. Cette technique revient à régulariser dans un certain sens la fonction marginale; il s'agira iei de régularisation par tranches relativement à une polarité.

Outre de nombreuses applications à la dualité quasiconvexe, cet approche va nous permettre d'obtenir de nouveaux exemples de problèmes d'optimisation en dualité comme les mesures de Jordan extérieure et intérieure d'un ensemble ou encore les intégrales de Riemann supérieure et inférieure d'une fonction bornée.

Soit donc $Z, X$ deux ensembles, $\varphi$ une fonction de $Z \times X$ dans $\overline{\mathbb{R}}, a \in Z$, et considérons le problème

$$
(P): \inf _{x \in X} \varphi(a, x) .
$$

On se donne aussi un ensemble $Y$ et une polarité $\Delta: 2^{Z} \rightarrow 2^{P}$. Le problème dual (Q) se définit en utilisant la fonction marginale $m \in \overline{\mathbb{R}}^{z}, m(z)=\inf _{x} \varphi(z, x)$, et en posant

$$
(Q): \sup _{y \notin \Delta a}-m^{4}(y)
$$

Si inf $(P)$ et sup $(Q)$ désignent les valeurs respectives de $(P)$ et $(Q)$ il vient done:

$$
\inf (P)=m(a) \geqslant m^{\Delta \Delta^{*}}(a)=\sup (Q) \text {. }
$$

Cette dualité s'inspire de [3], [25], [37], [39], ... avec la différence que notre problème dual est un problème avec contrainte; on obtient ainsi:

Proposimion IV.2.1. - L'ensemble des solutions optimales de $(Q)$ coïncide toujours avee $\partial^{\Delta} m^{\Delta \Delta^{*}}(a)$. Si $\inf (P)=\sup (Q)$, les solutions optimales de $(Q)$ coïncident avee $\partial^{4} m(a)$.

PREvVE. - $y$ solution de $(Q)$ équivaut d'après (28) à $y \notin \Delta a$ et $m^{\Delta \Delta^{*}}(a)=-m^{\Delta}(y)$ soit encore à $y \notin \Delta a$ et $m^{\Delta \Delta^{*}}(a)=-m^{\Delta \Delta^{*} A}(y)$ soit finalement à $y \in \partial^{\Delta} m^{\Delta \Delta^{*}}(a)$. En outre, $\inf (P)=\sup (Q)$ équivaut à $m(a)=m^{\Delta \Delta^{*}}(a)$ et il suffit d'appliquer prop. I.4.1 ii).

Considérons maintenant la fonction $L$ définie par:

$$
L: X \times Y \rightarrow \overline{\mathbb{R}}, \quad L(x, y)=\inf _{z \notin \Delta^{*} y} \varphi(z, x) \quad \text { si } y \notin \Delta a, \quad-\infty \quad \text { si } y \in \Delta a .
$$

Proposmion IV.2.2. - On a toujours $\sup (Q)=\sup _{y} \inf _{x} L(x, y)$, tandis que $\sup _{y} L(x, y)=\left(\varphi_{x}\right)^{\Delta \Delta^{*}}\left(\right.$ a) $\left(\right.$ avec $\left.\varphi_{x}(z)=\varphi(z, x)\right)$. 
Preuve. - Par définition de $L$ :

$$
\sup _{y} \inf _{x} L(x, y)=\sup _{y \neq \Delta a} \inf _{x} \inf _{z \neq \Delta^{*} y} \varphi(z, x)=\sup _{y \neq \Delta a, \inf _{z \notin \Delta^{*} y}} m(z)=m^{\Delta \Delta^{*}}(a)=\sup (Q) .
$$

D'autre part: $\sup _{y} L(x, y)=\sup _{y \notin \Delta a} \inf _{z \neq \Delta^{*} y} \varphi(z, x)=\varphi_{x}^{\Delta \Delta^{*}}(a)$.

On voit ainsi apparaître l'importance de la propriété:

$$
\text { Pour tout } \quad x \in X, \quad\left(\varphi_{x}\right)^{\Delta \Delta^{*}}(a)=\varphi_{x}(a) .
$$

Si $(\mathcal{T})$ a lieu on a en effet, d'après prop. IV.2.2, inf $(P)=\inf _{x} \sup _{y} L(x, y)$. Observons que $(\mathfrak{S})$ est vérifiée si, pour tout $x \in X$, les tranches de $\varphi_{x}$ sont fermées pour $\Delta^{*} \Delta$. Le théorème suivant montre alors que si les valeurs de $(P)$ et $(Q)$ coincident, les points selles de $L$ coïncident avec les couples solutions de $(P)$ et $(Q)$ et done que; pour la dualité par tranches, les approches lagrangienne et perturbationnelle sont équivalentes.

THÉoRÈne IV.2.3. - Si la propriété (S) a lieu, il y a équivalence entre

i) $\bar{x}$ solution de $(P), \bar{y}$ solution de $(Q), \inf (P)=\sup (Q) \in \mathbb{R}$;

ii) $(\bar{x}, \bar{y})$ est un point selle de $L$.

Prevve. - Pour i) $\Rightarrow$ ii) nous avons, d'après prop. IV.2.2, les inégalités inf $(P) \leqslant$ $\leqslant \sup _{y} L(\bar{x}, y)=\sup _{y \notin \Delta a} \inf _{z \notin \Delta^{*} y} \varphi(z, \bar{x}) \leqslant \varphi(a, \bar{x}) . \bar{x}$ étant solution de $(P)$, il vient alors $\inf (P)=$ $=\sup _{y} L(\bar{x}, y)$. D'autre part, d'après prop. IV.2.2,

$$
\begin{array}{rlrl}
\sup (Q) \leqslant \inf _{x} L(x, \bar{y}) & \leqslant \inf _{x} \inf _{z \notin \Delta^{*} \bar{y}} \varphi(z, x) & (\operatorname{car} \bar{y} \notin \Delta a) \\
& \leqslant \inf _{z \notin \Delta^{*} \bar{y}} m(z)=\sup (Q) & & (\text { car } \bar{y} \text { est solution de }(Q)) .
\end{array}
$$

Finalement, $\sup _{y} L(\bar{x}, y)=\inf (P)=\sup (Q)=\inf _{x} L(x, \bar{y}) \in \mathrm{R}$, autrement dit, $(\bar{x}, \bar{y})$ est un point selle de $L$.

Pour ii) $\Rightarrow$ i) l'on a $\sup (Q)=\inf _{x} L(x, \bar{y}) \in \mathrm{R}$, d'où il résulte que $\bar{y} \notin \Delta a$ et que $\sup (Q)=\inf _{z \neq \Delta^{*} \bar{y}} m(z)$ de sorte que $\bar{y}$ est bien solution de $(Q)$. De même, $\inf (P)=$ $=\sup _{y} L(\bar{x}, y)=\sup _{y \notin \Delta a} \inf _{z \notin \Delta^{*} y} \varphi(z, \bar{x})=\left(\varphi_{\bar{x}}\right)^{\Delta \Delta^{*}}(a)=\varphi(a, \bar{x})$ et $\bar{x}$ est bien solution de $(P)$.

IV.3. Problème d'optimisation posé par la mesure extérieure d'un ensemble.

Soit $\mu$ une fonction additive définie sur un clan $C$ de parties d'un ensemble $X$. On a donc, pour tous $A, B^{\prime} \in \mathcal{C}, A \cap B, A \cup B, A \backslash B \in \mathcal{C}$ ainsi que $\mu(A \cup B)=$ 
$=\mu(A)+\mu(B)$ si $A \cap B=\emptyset$. Soit $E \subset X$ et considérons le problème

$$
(P): \inf \{\mu(A) \mid A \in \mathcal{C}, A \supset E\}
$$

La fonction marginale associée à $(P)$ n'est autre que la fonction $\bar{\mu}$ mesure extérieure de Jordan des parties de $X$, ̀̀ savoir, pour tout $F \subset E, \vec{\mu}\left(F^{\prime}\right)=\inf \{\mu(A) \mid A \in \mathcal{C}$, $A \supset F\}$. Pour associer un dual au problème $(P)$ considérons la polarité $\Delta$ de $2^{2^{x}}$ dans $2^{\mathcal{C}}$ définie, pour tout $\mathcal{A} \subset 2^{x}$, par $\Delta \mathcal{A}=\left\{A \in \mathfrak{C} \mid F \in \mathcal{A} \Rightarrow A \cap F^{e} \neq \emptyset\right\}$. D'après $(28)$ le dual de $(P)$ relatif à $\Delta$ s'écrit

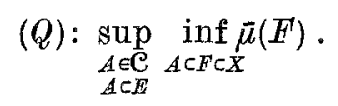

Sachant que $\bar{\mu}$ est une fonction croissante qui prolonge $\mu$, on obtient:

$$
(Q): \sup \{\mu(A) \mid A \in \mathcal{C}, A \subset E\} \text {. }
$$

Ainsi $(Q)$ représente la mesure de Jordan intérieure $\underline{\mu(E)}$ de $E$ et l'on voit que $\inf (P)=\sup (Q)$ si et seulement si $E$ est $\mu$-Jordan-intégrable.

Observons encore que $\bar{\mu}=\bar{\mu}^{\Delta \Delta^{*}}$ si et seulement si toutes les parties de $X$ sont $\mu$-Jordan-intégrables.

Proposimion IV.3.1. - Si $E$ est $\mu$-Jordan-intégrable, le A-quasidifférentiel de $\tilde{\mu}$ en $E$ coïncide aveo les éléments $A$ de $\mathcal{C}$ contenus dans $E$ et tels que $\mu(A)=\bar{\mu}(E)$.

Preuve. - On a ici $\sup (Q)=\inf (P)$ et, d'après prop. IV. 2.1., $\partial^{A} \tilde{\mu}(E)$ coïncide avec les solutions de $(Q)$, ce qui permet de conclure.

Pour calculer le lagrangien associé à $(P)$ et à $\Delta$, remarquons que $(P)$ s'écrit $\inf _{A \in \mathcal{C}} \varphi(E, A)$ où $\varphi$ est la fonction de $2^{x} \times \mathcal{C}$ dans $\overrightarrow{\mathbb{R}}$ définie $\operatorname{par} \varphi(F, A)=\mu(A)$ si $A \supset E,+\infty$ sinon. Le lagrangien $L$ défini par (30) prend ici la forme suivante:

$$
L: \mathbb{C} \times \mathfrak{C} \rightarrow \overline{\mathbb{R}}, L(A, B)= \begin{cases}-\infty & \text { si } B \cap E^{\mathfrak{c}} \neq \emptyset \\ \mu(A) & \text { si } B \subset E \text { et } B \subset A \\ +\infty & \text { si } B \subset E \text { et } R \notin A\end{cases}
$$

Par ailleurs, on vérifie que la propriété $(\mathfrak{F})$ a lieu si $E$ coïncide avec la réunion des éléments du clan $\mathcal{C}$ inclus dans $E$; ceci est vrai en particulier lorsque tous les points de $X$ sont dans $\mathcal{C}$, ce qui est le cas par exemple lorsque $\mathcal{C}$ est le clan des réunions finies de pavés de $\mathbb{R}^{n}$. Compte tenu des expressions de $(P)$ et $(Q)$, la prop. IV.2.2 et le théorème IV.2.3 donnent alors: 
THóorìne IV.3.2. - On a toujours $\underline{\mu}(E)=\sup _{A} \inf _{B} L(A, B)$. Si E est la réunion des étéments de $\mathrm{C}$ inclus dans $\Sigma, \vec{\mu}(E)$ s'écrit $\inf _{A} \sup _{B} L(A, B)$ et les points selles de $L$ coïncident alors avec les couples $(A, B) \in \mathcal{C}^{2}$ tels que $B \subset E \subset A$ et $\mu(A \backslash B)=0$.

ReMarque. - Pour ce qui est de l'intégrale supérieure au sens de Riemann d'une fonction bornée $f$ de $[a, b]$ dans $\mathbb{R}$, le problème s'écrit inf $\{I(g) \mid g \in \mathcal{E}$ et $g \geqslant f\}$ où $I: \mathcal{E} \rightarrow \mathbb{R}$ désigne l'intégrale des fonctions en escaliers. Il s'agit là d'un problème de programmation linéaire dans des espaces de dimensions infinies. Si l'on envisage d'associer un dual à ce problème en utilisant la dualité en programmation linéaire, on n'obtient rien d'intéressant. Par contre, si l'on considère la polarité $\Omega$ de $2^{\mathfrak{S}}$ dans $2^{\mathbb{E}}(\mathcal{B}$ désignant les fonctions réelles bornées sur $[a, b])$ définie par $\Delta A=\{g \in \mathbb{E} \mid h \in A \Rightarrow g \$ h\}$, on obtient pour dual l'intégrale de Riemann inférieure de $f$ sur $[a, b]$.

IV.4. Application a la dualité en programmation quasiconvexe.

Soit $(Z, Y)$ un couple d'e.l.e. mis en dualité par $\langle$,$\rangle ; on se donne un espace$ vectoriel $X$, une fonction $\varphi$ de $Z \times X$ dans $\overline{\mathbb{R}}$ et on considère le problème $(P): \inf _{x \in X} \varphi(0, x)$. Dans [7], Crouzetx introduit un dual dont la valeur égale celle de la régularisée quasiconvexe s.c.i. de la fonction marginale $m$ en 0 . Nous savons (cf. III.3 $b$ )) qu'une telle régularisation s'obtient en conjuguant par rapport à la polarité $\Delta: 2^{Z} \rightarrow 2^{\mathbf{Y} \times \mathbb{R}}$ avec $\Delta A=\{(y, r) \mid z \in A \Rightarrow\langle z, y\rangle \leqslant r\}$. Le dual de $(P)$ relatif à $\Delta$ s'éerit alors:

$$
(Q): \sup \left\{\inf _{\langle z, y\rangle>r} m(z) \mid y \in Y, r<0\right\} \text {. }
$$

On sait (prop. IV.2.1) que les solutions optimales de $(Q)$ coïncident avec $\partial^{\Delta} m^{\Delta \Delta^{*}}(0)$ qui est un cône "evenly" convexe épointé de $\bar{X} \times \mathbb{R}$ (Th. III.3.4). Le lagrangien introduit en (30) prend ici la forme

$$
L: X \times(Y \times \mathbb{R}) \rightarrow \mathbb{R}, \quad L(x,(y, r))=\inf _{\langle z, y\rangle>r} \varphi(z, x) \quad \text { si } r<0, \quad-\infty \quad \text { si } r \geqslant 0 .
$$

Les propositions IV.2.1, IV.2.2 et le théorème IV.2.3 permettent d'énoncer:

THÉOR亡̀Me IV.4.1. - On a toujours $\sup (Q)=\sup _{y, r} \inf _{x} L(x,(y, r))$. Si, pour tout $x \in X, \varphi(\cdot, x)$ est quasieonvexe s.c.i. alors inf $(P)=\inf _{\infty} \sup _{y, r} L(x ;(y, x))$ et les propriétés
suivantes sont équivalentes:

i) $\bar{x}$ solution de $(P),(\bar{y}, \bar{r})$ solution de $(Q), \inf (P)=\sup (Q) \in \mathbb{R}$;

ii) $(\bar{x},(\bar{y}, \bar{r}))$ est un point selle de $L$.

RFMaRque. - En dualité quasiconvexe s.c.i. on ne connaissait pas, jusqu'à présent, de lagrangien donnant l'équivalence entre i) et ii). 
Dans [35], [36], Passt et Prismax considèrent des problèmes du type inf $\varphi(0,0, x)$ où $\varphi: Z \times \mathbb{R} \times X \rightarrow \overline{\mathbb{R}}$ et où $(Z, Y)$ et $(X, U)$ sont des couples d'e.l.c. en dualité. Nous obtenons exactement leur problème dual et leur lagrangien en considérant la polarité $\Omega$ de $2^{Z \times \mathbb{R}}$ dans $2^{Y \times \mathbb{R}}$ avec $\Omega A=\{(y, s) \mid(z, r) \in A \Rightarrow\langle z, y\rangle+r s<0\}$. Les vecteurs de K.T. qu'ils introduisent à cette occasion coïncident avec le $\Delta$-quasidifférentiel de $m$ en $(0,0)$ où $m(z, r)=\inf _{x} \varphi(z, r, x)$. Ils envisagent également des problèmes du type $\inf _{x, r} \psi(0, x, r)$ où $\psi: Z \times X \times \mathbb{R} \rightarrow \overline{\mathbb{R}}$. Nous récupérons exactement leur problème dual et leur lagrangien en considérant la polarité $\Sigma$ de $2^{Z}$ dans $2^{P}$ avec $\Sigma A=\{y \mid z \in A \Rightarrow\langle z, y\rangle\langle 0\} ;$ les vecteurs de K.T. définis dans [35] coïncident alors avec notre $\Sigma$-quasidifférentiel de $m$ en 0 où $m(z)=\inf _{x, r} \psi(z, x, r)$. Le théorème 4.2 de [35] est alors une conséquence du théorème IV.2.3.

Je tiens à remercier le referee dont j’ai beaucoup apprécié la critique constructive.

\section{REFERENCES}

[1] J. ARAOZ - J. EDMONDS - V. J. GRIFFIN, Polarities given by systems of bilinear inequalities, Math. Op. Res., vol. 8, n. 1 (1983), pp. 34-41.

[2] M. AtThia - A. El Qortobi, Quasiconvex duality, Lecture Notes in Control, 30, Springer Verlag (1981), pp. 3-8.

[3] S. J. BALDER, An extension of duality stability relations to non convex optimization problems, Siam, J. Contr. Opt., 15 (1977), pp. 329-343.

[4] G. Birkнoff, Lattice theory, Providence, A.M.S., 1966.

[5] Y. Chabril.tac, Thèse de 3-ème eycle, Clermont II, 1982.

[6] G. ChoфUet, Sur les notions de filtre et de gritte, C.R.A.S. Paris, 884 (1947), pp. 171-173.

[7] J. P. Crovzeiz, Contribution à l'étude des fonctions quasiconvexes, Thèse, Clermont II, 1977.

[8] J. P. Crovzerx, Duality between direct and indirect utility functions, J. Math. Eco., vol. 12, n. 2 (1983), pp. 139-165.

[9] S. DoLECKI, Remarks on semicontinuity, Bull. Ac. Pol. Ser. Math., 85 (1977), pp. 863-867.

[10] S. DoLeckI, Abstract study of optimality conditions, J. Math. Anal. Appl., 73 (1980), pp. 24-58.

[11] S. DoLtekr - G. Greco, Oyrtologies and convergence, I, mimeo Université de Trente. 1983 .

[12] DURIER, Communication personnelle.

[13] A. EL QortoвI, These de 3-ème cycle, Toulouse, 1980.

[14] K. H. ELSTER - R. NeHSE, Zur theorie der polarfunktionale, Math. Oper. forsch. Stat., 5 (1974), pp. 3-21.

[15] C. J. Everetr, Closure operators and Gatois theory in lattices, Trans. Amer. Math. Soc., vol. 55 (1944), pp. 514-525.

[16] J. J. M. EVERS - H. V. MAAREN, Duality principles in mathematics and their relations to conjugate functions, Dpt of applied mathematics, Twente Univ. of Teeh., memorandum $336(1981)$.

[17] W. FencheL, On eonjugate convex functions, Can. J. Math., 1 (1949), pp. 73-77.

[18] W. Fenchel, Oonvex cones, sets and functions, mimeo Lectures Notes, Princeton University, 1951. 
[19] J. FLACHS - POLLATSCHEK, Duality theorems for certain programs involving minimum or maximum operations, Math. Prog., 16 (1979), pp. 348-370.

[20] J. FxaChs, Global saddle point duality for quasiconcave programs, Math. Prog., 80 (1981), pp. 327-347.

[21] J. FLACHS, Global saddle point duality for quasiconcave programs, II, Math. Prog., 84 (1982), pp. 326-345.

[22] H. J. Greenberg - W. P. PIERskalla, Quasieonjugate functions and surrogate duality, Cahier Centre Etude, Rech. Oper., 15 (1973), pp. 437-448.

[23] E. H. IVANOF - R. NeHsE, Relations between generalized concepts of convexity and conjugacy, Math. Oper. Stat. Ser. Opt., 13, vol. 1 (1982), pp. 9.18.

[24] S. S. KuKateladZE - A. M. Rubinov, Minkowski's duality and its applications, Nauka, Novosibirsk, 1976.

[25] P. O. Lindberg, A generalization of Fenchel conjugation, Proc. IX Int. Symp. on Math. Prog., vol. 2, Akad Kiado Budapest, 1979.

[26] J. E. Martinez Legaz, Exact quasiconvex conjugation, Symp. Math. Prog., Bonn, 1982.

[27] J. E. Martinez Legaz, A new approach to symmetric quasiconvex conjugacy, Sym. Oper. Res. Karlsruhe, 1983.

[28] J. E. MARTinez LEGaz, Conjugacion associada a un grafo, Jornadas Matematicas HispanoLusas, 1982.

[29] J. J. Moreat, Fonctionnelles convexes, Collège de France, 1966.

[30] J. J. MoReat, Inf convolution, sous additivité, convexité des fonctions numériques, J. Math. Pures. Appl., 49 (1970), pp. 109-154.

[31] E. H. Moore, Introduction to a form of general analysis, Amer. Math. Soc. Coll. Pub., vol. 2, (1910).

[32] 0. Ore, Galois connexions, Trans. Amer. Math. Soc., vol. 55 (1944), pp. 493-513.

[33] O. ORE, Theory of graphs, A.M.S., coll. Pub., vol. XXXVIII, (1962).

[34] U. Passy - E. Z. Prisman, On quasiconvex functions and their conjugate, mimeo 297, Fac. of Ind. and Mang. Eng. Technion Haifa Israel, 1981.

[35] U. Passy - E. Z. Prisman, Duality in quasiconvex programming, mimeo, 308 (1981), pp. $123-456$.

[36] U. Passt - E. Z. Prisman, Saddle functions and min-max problems, mimeo, 303 (1981), pp. $123-456$.

[37] J. P. PeNot - M. VoLLe, On quasiconvex duality, en préparation.

[38] G. PICKERT, Bemerkungen über Galois-Verbindungen, Arch. Math., vol. 3 (1952), pp. 285-289.

[39] R. T. Rockafellar, Convex analysis, Princeton, 1970.

[40] I. Sirger, Pseudoconjugate functionals and pseudoduality, Math. Meth. Op. Res. Sofia, (1981), pp. 115-134.

[41] M. VoLLE, Multiapplications duales et problèmes d'optimisation en dualité, C.R.A.S.Paris, 896 (1983), pp. 11-15.

Note ajoutée en épreuves. - Depuis que cet article a été soumis, j'ai eu connaissance des récents travaux suivants où, dans un cadre vectoriel et géométrique, des dualités de substitution sout étudiées qui font jouer un rôle essential aux tranches des fonctions:

I. SINGER, The lower semi-continuous quasiconvex hull as a normatized second conjugate, Non linear Anal., 7, no. 10 (1983), pp. 1115-1121.

I. SINGER, Surrogate conjugate functionals and surrogate convexity, Applicable AnaI., 16 (1983), pp. 291-327.

I. SINGer, Conjugate functionals and level sets, Non linear Anal., 8 (1984), pp. 313-320. 